\title{
Physical parameters and wind properties of galactic early B supergiants
}

\author{
P. A. Crowther ${ }^{1}$, D. J. Lennon ${ }^{2}$, and N. R. Walborn ${ }^{3}$ \\ 1 Department of Physics and Astronomy, University of Sheffield, Hicks Building, Hounsfield Rd, Shefffield, S3 7RH, UK \\ e-mail: Paul.Crowther@sheffield.ac.uk \\ 2 Isaac Newton Group, Apartado 321, 38700 Santa Cruz de La Palma, Canary Islands, Spain \\ 3 Space Telescope Science Institute, 3700 San Martin Drive, Baltimore MD 21218, USA
}

Received 23 June 2005 / Accepted 12 September 2005

\section{ABSTRACT}

We present optical studies of the physical and wind properties, plus CNO chemical abundances, of 25 O9.5-B3 Galactic supergiants. We employ non-LTE, line blanketed, extended model atmospheres, which provide a modest downward revision in the effective temperature scale of early B supergiants of up to 1-2 kK relative to previous non-blanketed results. The so-called "bistability jump" at B1 ( $\left.T_{\text {eff }} \sim 21 \mathrm{kK}\right)$ from Lamers et al. is rather a more gradual trend (with large scatter) from $v_{\infty} / v_{\text {esc }} \sim 3.4$ for B0-0.5 supergiants above $24 \mathrm{kK}$ to $v_{\infty} / v_{\text {esc }} \sim 2.5$ for B0.7-1 supergiants with $20 \mathrm{kK} \leq T_{\text {eff }} \leq 24 \mathrm{kK}$, and $v_{\infty} / v_{\text {esc }} \sim 1.9$ for B1.5-3 supergiants below $20 \mathrm{kK}$. This, in part, explains the break in observed UV spectral characteristics between B0.5 and B0.7 subtypes as discussed by Walborn et al. We compare derived (homogeneous) wind densities with recent results for Magellanic Cloud B supergiants and generally confirm theoretical expectations for stronger winds amongst Galactic supergiants. However, winds are substantially weaker than predictions from current radiatively driven wind theory, especially at midB subtypes, a problem which is exacerbated if winds are already clumped in the $\mathrm{H} \alpha$ line forming region. In general, CNO elemental abundances reveal strongly processed material at the surface of Galactic B supergiants, with mean N/C and N/O abundances 10 and 5 times higher than the Solar value, respectively, with HD 2905 (BC0.7 Ia) indicating the lowest degree of processing in our sample, and HD 152236 (B1.5 Ia ${ }^{+}$) the highest.

Key words. stars: early-type - stars: fundamental parameters - stars: abundances - stars: evolution - stars: mass-loss

\section{Introduction}

Early-type stars possess powerful winds that provide significant feedback to the local ISM (e.g. Smith 2005). Significant progress has recently been made towards an improved understanding of the physical parameters and winds of massive $\mathrm{O}$ stars and their immediate descendants, OBA supergiants. In particular, the availability of modern spectral synthesis codes allowing for line blanketing and stellar winds together with high-resolution instruments on 4-8 $\mathrm{m}$ telescopes permits detailed studies of $\mathrm{OB}$ stars in the Magellanic Clouds (e.g. Crowther et al. 2002; Hillier et al. 2003; Trundle et al. 2004). A primary motive behind such studies is the investigation of how winds in the low-metallicity LMC and SMC differ from those in the Milky Way.

For O stars, Herrero et al. (2003) and Repolust et al. (2004) provide suitable Galactic comparison stars, whilst one currently has to rely on results from Kudritzki et al. (1999) for B supergiants, for which the temperature scale of McErlean et al. (1999) was adopted. This is one of the principal reasons behind the present study, namely a determination of the wind properties and temperature scale of $\mathrm{B} 0-3$ supergiants using contemporary line blanketed techniques and optical spectroscopy. Although the range in spectral type is narrow, the effective temperatures of $\mathrm{B} 0-3$ supergiants span almost a factor of $t w o$, from 17 to $32 \mathrm{kK}$ (McErlean et al. 1999). The present optical study of ionization in early B supergiants complements the recent UV wind ionization investigation by Prinja et al. (2005). Further, there is now considerable qualitative evidence supporting CNO-processed material in the atmospheres of massive stars (Herrero \& Lennon 2004), although quantitative results for B supergiants - for comparison with recent evolutionary models allowing for rotation (Meynet \& Maeder 2000) - are generally lacking.

A second motive for the present study is an observational investigation of the so-called "bistability jump" in the terminal velocities of early B supergiants (Lamers et al. 1995). Walborn \& Nichols-Bohlin (1987) first remarked upon the UV morphological differences between B0.5 Ia and B0.7 Ia supergiants, in the sense that C II $\lambda 1335$ and Al III $\lambda 1855-63$ P Cygni profiles first appear at B0.7, whilst the Si IV $\lambda 1393-1402$ doublet becomes narrower and stronger. In solely two cases, HD 115842 (B0.5 Ia) and HD 91943 (B0.7 Ia) are these distinctions reversed (Walborn et al. 1995). Pauldrach \& Puls (1990) first 
Table 1. Program Stars - observations are from either the 1m JKT (Lennon et al. 1992), $2.5 \mathrm{~m}$ INT or $4.2 \mathrm{~m}$ WHT (northern) or $1.5 \mathrm{~m}$ CTIO (southern). Two estimates of interstellar reddenings are given, based on intrinsic colours adopted from Schmidt-Kaler (1982) and from theoretical continuum fits to UV spectrophotometry and optical photometry (no spectrophotometry was available for HD 194279 or HD 148688). The majority of our targets are probable cluster/association members, whilst absolute magnitudes are adopted for the remainder, based on average values of Magellanic Cloud members from Fitzpatrick (1991), Parker (1993) and Massey et al. (1995), except that HD 190603 is set at $M_{V}=-7.5$ mag. References to cluster/association distances are: (a) Brown et al. (1994); (b) Blaha \& Humphreys (1989); (c) Baume et al. 1999; (d) Baume et al (2003); (e) Garmany \& Stencel (1992); (f) Humphreys (1978).

\begin{tabular}{|c|c|c|c|c|c|c|c|c|c|c|c|}
\hline HD & Name & Sp type & $\begin{array}{l}V \\
\text { mag }\end{array}$ & $\begin{array}{l}B-V \\
\text { mag }\end{array}$ & $\begin{array}{r}E(B \\
(B-V)_{0}\end{array}$ & $\begin{array}{l}-V) \\
\mathrm{UV}\end{array}$ & $\begin{array}{l}\text { Assoc. } \\
\text { mag }\end{array}$ & $\mathrm{DM}$ & $\begin{array}{l}\text { Ref } \\
\text { mag }\end{array}$ & $\begin{array}{l}M_{V} \Delta M_{V} \\
\text { mag }\end{array}$ & Obs \\
\hline 30614 & $\alpha \mathrm{Cam}$ & O9.5 Ia & 4.29 & +0.02 & 0.29 & 0.32 & & 10.0 & & $-6.6 \pm 0.5$ & WHT \\
\hline 37128 & $\epsilon$ Ori & B0 Ia & 1.70 & -0.19 & 0.05 & 0.06 & Ori OB1b & 7.8 & $\mathrm{a}$ & $-6.3 \pm 0.5$ & WHT/INT \\
\hline 91969 & & B0 Ia & 6.52 & +0.00 & 0.24 & 0.26 & NGC3293 & 12.2 & d & $-6.4 \pm 0.3$ & CTIO \\
\hline 94909 & & B0 Ia & 7.34 & +0.48 & 0.72 & $0.75 \neq$ & & 11.5 & & $-6.4 \pm 0.5$ & CTIO \\
\hline 122879 & & B0 Ia & 6.42 & +0.12 & 0.36 & 0.37 & & 11.7 & & $-6.4 \pm 0.5$ & CTIO \\
\hline 38771 & $\kappa$ Ori & B0.5 Ia & 2.05 & -0.18 & 0.04 & 0.07 & Ori OB1c & 8.0 & a & $-6.1 \pm 0.5$ & WHT/INT \\
\hline 115842 & & B0.5 Ia & 6.02 & +0.29 & 0.51 & $0.57 \ddagger$ & & 11.3 & & $-6.9 \pm 0.5$ & CTIO \\
\hline 152234 & & B0.5 Ia (N wk) & 5.44 & +0.20 & 0.42 & 0.45 & Sco OB1 & 11.5 & $\mathrm{c}$ & $-7.4 \pm 0.5$ & CTIO \\
\hline 2905 & $\kappa \mathrm{Cas}$ & BC0.7 Ia & 4.16 & +0.14 & 0.35 & 0.39 & Cas OB14 & 10.2 & $\mathrm{~b}$ & $-7.1 \pm 0.3$ & WHT/INT \\
\hline 91943 & & B0.7 Ia & 6.73 & +0.07 & 0.28 & $0.22 \ddagger$ & NGC 3293 & 12.2 & d & $-6.3 \pm 0.3$ & CTIO \\
\hline 152235 & & B0.7 Ia (N wk) & 6.34 & +0.51 & 0.72 & $0.75 \ddagger$ & Sco OB1 & 11.5 & $\mathrm{c}$ & $-7.4 \pm 0.5$ & CTIO \\
\hline 154090 & & B0.7 Ia & 4.87 & +0.26 & 0.47 & $0.49 \ddagger$ & & 10.2 & & $-6.8 \pm 0.5$ & CTIO \\
\hline 13854 & & B1 Iab & 6.48 & +0.28 & 0.47 & 0.55 & Per OB1 & 11.8 & $\mathrm{e}$ & $-6.8 \pm 0.5$ & WHT/INT \\
\hline 91316 & $\rho$ Leo & B1 Iab (N str) & 3.85 & -0.14 & 0.05 & 0.06 & & 10.5 & & $-6.8 \pm 0.5$ & CTIO \\
\hline 148688 & & B1 Ia & 5.33 & +0.33 & 0.52 & $0.52 \ddagger$ & & 10.5 & & $-6.8 \pm 0.5$ & CTIO \\
\hline 14956 & & B $1.5 \mathrm{Ia}$ & 7.19 & +0.72 & 0.90 & $0.95 \ddagger$ & Per OB1 & 11.8 & e & $-7.4 \pm 0.5$ & WHT/INT \\
\hline 152236 & $\zeta^{1} \mathrm{Sco}$ & $\mathrm{B} 1.5 \mathrm{Ia}^{+}$ & 4.73 & +0.48 & 0.66 & 0.69 & Sco OB1 & 11.5 & $\mathrm{c}$ & $-8.8 \pm 0.5$ & CTIO \\
\hline 190603 & & $\mathrm{~B} 1.5 \mathrm{Ia}^{+}$ & 5.65 & +0.54 & 0.72 & $0.80 \ddagger$ & & 10.9 & & $-7.5 \pm 1.0$ & WHT/INT \\
\hline 14143 & & B2 Ia & 6.66 & +0.50 & 0.67 & 0.70 & $h$ Per & 11.8 & $\mathrm{e}$ & $-7.2 \pm 0.3$ & WHT/INT \\
\hline 14818 & 10 Per & B2 Ia & 6.25 & +0.30 & 0.47 & 0.52 & Per OB1 & 11.8 & $\mathrm{e}$ & $-7.0 \pm 0.5$ & WHT/INT \\
\hline 41117 & $\chi^{2}$ Ori & B2 Ia & 4.63 & +0.28 & 0.45 & 0.48 & Gem OB1 & 10.9 & $\mathrm{~b}$ & $-7.6 \pm 0.3$ & WHT \\
\hline 194279 & & B2 Ia & 7.05 & +1.02 & 1.19 & $1.26 \%$ & Cyg OB9 & 10.4 & $\mathrm{f}$ & $-7.0 \pm 0.3$ & WHT/INT \\
\hline 198478 & 55 Cyg & B2.5 Ia & 4.86 & +0.42 & 0.57 & 0.59 & Cyg OB7 & 9.5 & $\mathrm{f}$ & $-6.4 \pm 0.3$ & WHT \\
\hline 14134 & & B3 Ia & 6.55 & +0.45 & 0.58 & $0.65 \ddagger$ & $h$ Per & 11.8 & $\mathrm{e}$ & $-7.1 \pm 0.3$ & JKT/INT \\
\hline 53138 & $o^{2} \mathrm{CMa}$ & B3 Ia & 3.01 & -0.08 & 0.05 & 0.07 & & 10.2 & & $-7.3 \pm 0.5$ & JKT/INT \\
\hline
\end{tabular}

$\$$ : UV spectrophotometry absent or poor quality, leading to uncertain reddening from this technique.

described a bi-stability jump with reference to the extreme B supergiant $\mathrm{P}$ Cygni $\left(\mathrm{B} 1.5 \mathrm{Ia}^{+}\right)$, in the sense that a small change in physical properties for $\mathrm{P}$ Cyg can result in dramatic differences in the outer wind, either ionized or recombined (Najarro et al. 1997; Crowther 1997). Amongst normal B supergiants, with much weaker winds, Lamers et al. (1995) demonstrated that the ratio $v_{\infty} / v_{\text {esc }}$ drops steeply from about 2.6 for temperatures above $21 \mathrm{kK}$ (B1 subtype), to a value of 1.3 below ${ }^{1}$.

The empirical B supergiant bistability jump of Lamers et al. has been widely used in synthetic spectral model calculations, of application to distant star forming galaxies (e.g. Rix et al. 2004). Vink et al. $(1999,2000)$ predicted a jump in massloss around $25 \mathrm{kK}$, due to the line acceleration of Fe III, such

\footnotetext{
${ }^{1}$ Prinja \& Massa (1998) suggested a larger observational spread in $v_{\infty} / v_{\text {esc }}$ amongst early B supergiants.
}

that the mass-loss increases by a factor of 5 between $27.5 \mathrm{kK}$ and $22.5 \mathrm{kK}$, although the change in velocity was not thoroughly explained. Here, we present studies of B supergiants to investigate the Lamers et al. bistability jump, and test whether the Vink et al. predictions are consistent with observations.

\section{Observations}

We have selected 25 Galactic early-type supergiants in the spectral range $\mathrm{O} 9.5-\mathrm{B} 3,75 \%$ of which have established cluster/association membership, listed in Table 1. In all but two cases, robust UV wind velocities are available from the literature - otherwise average subtype values from Howarth et al. are adopted (HD 19456, HD 192479). Cluster or association distance estimates are available for the majority of the sample, whilst average subtype absolute magnitudes are adopted 
for the remainder based on Magellanic Cloud stars (Fitzpatrick 1991; Parker 1993; Massey et al. 1995). Many of the associations are rather extended (e.g. Per OB1: Kendall et al. 1995) so in general we adopt uncertainties of \pm 0.5 mag in distance modulus/absolute magnitude for field and association members. Reduced uncertainties of \pm 0.3 mag are restricted to cluster members (NGC 3293, $h$ Per) and the more spatially concentrated OB associations (Cyg OB7, Cyg OB9, Gem OB1, Cas OB14). For the two Ori OB1 members, we adopt distances from individual Ori OB1 subgroups from Brown et al. (1994), Ib for HD 37128 and Ic for HD 38771. The latter distance of $400 \mathrm{pc}$ is in preference to $220 \mathrm{pc}$ as derived from Hipparcos, given the difficulty in determining individual distance estimates along this line of sight (de Zeeuw et al. 1999). For HD 190603, we adopt an absolute magnitude of $-7.5 \pm 1.0 \mathrm{mag}$, intermediate between normal Ia supergiants and the hypergiant HD 152236. Finally, we consider the Hipparcos membership of HD 53138 to Col. 121 to be dubious since it has insignificant proper motion compared to the cluster (de Zeeuw et al. 1999), and so instead adopt an average B3 Ia absolute magnitude.

Interstellar reddenings (assuming $R=A_{\mathrm{V}} / E(B-V)=$ 3.1 throughout) are determined using intrinsic colours from Schmidt-Kaler (1982) and independently from theoretical flux distributions determined in the present analysis, together with archival large aperture $I U E$ spectrophotometry (except for HD 194279 and HD 148688). Although the former was adopted for distance modulus/absolute magnitude estimates, the latter provided a check on the reliability of intrinsic colours for early B supergiants. In general the combined UV/optical flux distribution suggested intrinsic $(B-V)_{0}$ values $\sim 0.02 \mathrm{mag}$ more negative than Schmidt-Kaler (1982) for our Ia sample.

Optical observations originate from either the Isaac Newton Group, La Palma (northern sample) or CTIO, Chile (southern sample). The northern targets were originally observed in the blue visual with the JKT (Lennon et al. 1992) with the Richardson-Brealey Spectrograph with the R1200B grating at a resolution of $0.8 \AA$ and $S / N \sim 150$. The majority were re-observed with the ISIS spectrograph at the WHT at a similar resolution (Smartt, priv. comm.) though higher $S / N(\geq 300)$ using a setup identical to that used in Smartt et al. (2001). $\mathrm{H} \alpha$ observations were obtained at the INT using the IDS spectrograph by DJL, with a spectral resolution of $1.2 \AA$, and $S / N \sim 200$. In three cases, HD 30614, HD 41117 and HD 198478, WHT ISIS observations were obtained by PAC during 2002 October using a dichroic and 1.2 arcsec wide slit, providing simultaneous blue and $\mathrm{H} \alpha$ spectroscopy with the $1200 B / R$ gratings in first order, centred at $\lambda 4400$ and $\lambda 6650$, with $S / N \geq 300$.

The southern sample was observed at the CTIO $1.5 \mathrm{~m}$ during May-June 2003 via the SMARTS service program using the Cassegrain spectrograph with Loral $1 \mathrm{~K}(1200 \times$ 800 pixel) CCD. A standard reduction, bias subtraction, flatfielding, and extraction were carried out using IRAF. Blue and red spectroscopy of each target was obtained using the 47 grating, providing a spectral range, and (3 pixel) spectral resolution of $660 \AA, 1.6 \AA$ (second order, blue) and $1320 \AA 3.0 \AA$ (first order, red) respectively, as measured from $\mathrm{HeAr}$ and $\mathrm{Ne}$ arc lines.
The extracted blue and red continuum $S / N$ ranges from 150 to 200 , and 200 to 300 respectively.

In order to assess the robustness of our analyses based on intermediate resolution observations covering a restricted wavelength range, HD 115842 was observed for us by Dr C.J. Evans (ESO programme 073.D-0234) at the MPG/ESO $2.2 \mathrm{~m}$ on 7 July 2004 with the Fibre-fed, Extended Range Echelle Spectrograph (FEROS). A two arcsec aperture provides a resolving power of $R=48000$ covering a spectral range of 3500-9200 $\AA$ using an EEV $2 \mathrm{k} \times 4 \mathrm{k}$ CCD. A single exposure of $240 \mathrm{~s}$ provided a continuum $S / N$ of 300 at $\lambda 4600$ in the pipeline reduced dataset (A. Kaufer, priv. comm.).

Finally, (at least) two of the present sample definitely possess significant $\mathrm{H} \alpha$ variability, HD 37128 (B0 Ia, Prinja et al. 2004) and HD 14134 (B3 Ia, Morel et al. 2004). For HD 37128 we investigate the effect of variability on the derived mass-loss rates using $\mathrm{H} \alpha$ observations provided by Dr R.K. Prinja.

\section{Analysis}

\subsection{Methods}

Recent developments in model-atmosphere studies of hot stars routinely permit sophisticated metal-line blanketing in nonLTE to be considered, under either plane-parallel geometry (e.g., TLUSTY: Hubeny \& Lanz 1995; Lanz \& Hubeny 2003) or spherical geometry (e.g., CMFGEN: Hillier \& Miller 1998). The former technique provides a more comprehensive treatment of photospheric metal-line blanketing, while the latter offers insights into wind properties.

In the present application, we have utilized CMFGEN, the current version of which is discussed by Hillier et al. (2003). The code solves the radiative transfer equation in the comoving frame, under the additional constraints of radiative and statistical equilibrium. The model used here is similar to that applied by Evans et al. (2004b). In total, 26 ions of H, He, C, N, $\mathrm{O}, \mathrm{Mg}, \mathrm{Al}, \mathrm{Si}, \mathrm{S}, \mathrm{Ca}, \mathrm{Fe}$ are included for early subtypes, comprising a total of 2636 individual levels (grouped into $766 \mathrm{su-}$ perlevels), with a full array of 25960 bound-bound transitions. Higher ionization stages are omitted for B2.5-3 supergiants as indicated in Table 2.

The temperature structure is determined by radiative equilibrium. CMFGEN does not solve the momentum equation, so that a density or velocity structure is required. For the supersonic part, the velocity is parameterized with a classical $\beta$-type law, with the exponent in the range $\beta=1-3$ selected on the basis of the $\mathrm{H} \alpha$ profile. This is connected to a hydrostatic density structure at depth, such that the velocity and velocity gradient match at the interface. The subsonic velocity structure is set by the corresponding H-He TLUSTY model which were calculated by the authors for each CMFGEN model atmosphere calculation. With regard to CMFGEN models in which the TLUSTY structure at depth is neglected, stellar temperatures are $\sim 1 \mathrm{kK}$ lower and mass-loss rates $0.1-0.2$ dex higher.

We initially estimated surface gravities at individual temperatures from a 2 nd order fit to results from Trundle et al. (2004) and Trundle \& Lennon (2005) for Ia B supergiants, plus HD 30614 (O9.5 Ia) from Repolust et al. (2004). This is presented in Fig. 1 and should lead to gravities accurate 
Table 2. Atomic models for early and mid B supergiants. For each ion F denotes full levels, S super levels, and T the number of bound-bound transitions considered. Most ions are common to all stars, except that high ionization stages are only included in early B supergiant models (shown in italics).

\begin{tabular}{|c|c|c|c|c|c|c|c|c|c|c|c|c|c|c|}
\hline \multicolumn{3}{|c|}{ Element I } & \multicolumn{3}{|c|}{ II } & \multicolumn{3}{|c|}{ III } & \multicolumn{3}{|c|}{ IV } & \multicolumn{3}{|c|}{ V } \\
\hline & $\mathrm{F} S$ & $\mathrm{~T}$ & $\mathrm{~F}$ & S & $\mathrm{T}$ & $\mathrm{F}$ & S & $\mathrm{T}$ & $\mathrm{F}$ & S & $\mathrm{T}$ & $\mathrm{F}$ & S & $\mathrm{T}$ \\
\hline $\mathrm{H}$ & 3020 & 435 & & & & & & & & & & & & \\
\hline $\mathrm{He}$ & 5941 & 590 & 30 & 20 & 435 & & & & & & & & & \\
\hline $\mathrm{C}$ & & & 53 & 30 & 323 & 54 & 29 & 268 & 18 & 13 & 76 & & & \\
\hline $\mathrm{N}$ & 2210 & 59 & 41 & 21 & 144 & 70 & 34 & 430 & & & & & & \\
\hline $\mathrm{O}$ & 7518 & 450 & 63 & 22 & 444 & 45 & 25 & 182 & & & & & & \\
\hline $\mathrm{Mg}$ & & & 45 & 18 & 362 & & & & & & & & & \\
\hline $\mathrm{Al}$ & & & 44 & 26 & 171 & 65 & 21 & 1452 & & & & & & \\
\hline $\mathrm{Si}$ & & & 62 & 23 & 365 & 45 & 25 & 172 & 12 & 8 & 26 & & & \\
\hline S & & & 87 & 27 & 786 & 41 & 21 & 177 & 92 & 37 & 708 & & & \\
\hline $\mathrm{Ca}$ & & & 12 & 7 & 28 & & & & & & & & & \\
\hline $\mathrm{Fe}$ & & & 510 & 100 & 7501 & 607 & 655 & 5482 & 272 & 483 & 3113 & 182 & 46 & 1781 \\
\hline
\end{tabular}

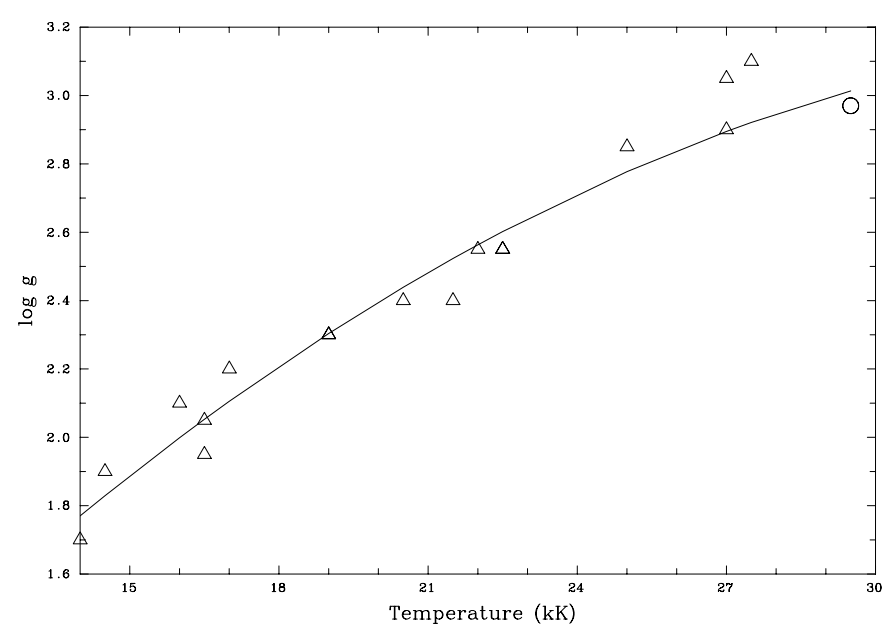

Fig. 1. $\log g-T_{\text {eff }}$ calibration from Trundle et al. (2004) and Trundle \& Lennon (2005) for SMC B supergiants (open triangles) plus HD 30614 from Repolust et al. (2005, open circle).

to \pm 0.2 dex. Subsequently, we have adjusted surface gravities in TLUSTY models until $\mathrm{H} \gamma$ is reproduced. We anticipate accuracies of $0.1-0.15$ dex for all targets except perhaps the hypergiants HD 152236 and HD 190603 which have significant wind contamination at $\mathrm{H} \gamma$.

We have assumed a depth-independent Doppler profile for all lines when solving for the atmospheric structure in the comoving frame, while in the final calculation of the emergent spectrum in the observer's frame, we have adopted a radially dependent turbulence ${ }^{2}$, which reflects the effect of shocks due to wind instabilities. For the present sample we initially assume $\xi=20 \mathrm{~km} \mathrm{~s}^{-1}$, with other values in the range $10-40 \mathrm{~km} \mathrm{~s}^{-1}$ considered if consistent fits are not obtained for the helium and silicon lines. Incoherent electron scattering and Stark broadening for hydrogen and helium lines are adopted. We convolve

\footnotetext{
${ }^{2}$ In CMFGEN this adopted value is technically $\xi_{\min }$, the microturbulence at the base of the wind, which increases with radius to $\xi_{\max }=50 \mathrm{~km} \mathrm{~s}^{-1}$ at $v_{\infty}$.
}

our synthetic spectrum with a rotational broadening profile. Because of the intermediate dispersion of most observations, instrumental effects prevent a reliable determination of $v \sin i$ from the present dataset, such that we adopt the UV derived rotational rates from Howarth et al. (1997).

Terminal velocities, $v_{\infty}$, were uniformly determined from $v_{\text {black }}$ measurements from the literature (Howarth et al. 1997), and are listed in Table 4. Alternatively, velocities may be derived via the Sobolev with Exact Integral (SEI) technique (Lamers et al. 1987; Haser 1995). Although individual measurements differ by as much as $20 \%, v_{\infty}$ (Haser) $/ v_{\infty}$ (Howarth et al.) $=1.02$ for the 19 stars in common to both samples. SEI modelling is often preferred to $v_{\text {black }}$ measurements of wind velocity, although has its own modelling uncertainties. For example, Haser (1995) and Evans et al. (2004a) both used SEI modelling of UV lines in HD 152236, deriving $v_{\infty}=$ $500 \mathrm{~km} \mathrm{~s}^{-1}$ and $450 \mathrm{~km} \mathrm{~s}^{-1}$, respectively, versus $390 \mathrm{~km} \mathrm{~s}^{-1}$ adopted here from Howarth et al. (1997).

We varied the mass-loss rate and the velocity law (as characterized by the exponent $\beta$ ) until the $\mathrm{H} \alpha$ profile was best reproduced in terms of intensity and morphology. Next, we adjusted the stellar temperature to match the intensities of the He II $\lambda 4542$ and He I $\lambda 4471$ lines for O9.5-B0 stars, Si IV $\lambda 4089$ and Si III $\lambda 4553-68-75$ lines for B0.5-B2 stars, and Si II $\lambda 4128-31$ and Si III $\lambda 4553-68-75$ lines for B2.5-3 subtypes. The accuracy with which we can derive stellar temperatures is $\pm 1000 \mathrm{~K}$. Radii and luminosities follow from our derived absolute magnitude, whilst mass-loss rates are obtained from a spectral fit to the $\mathrm{H} \alpha$ profile. With absolute magnitudes fixed at our adopted values, formal uncertainties of $10 \%$ in $\mathrm{d} M / \mathrm{d} t$ follow for stars with strong winds, increasing to $30 \%$ for weak lined stars. In general, the greatest uncertainty relates to the $\beta$ law, although the $\mathrm{H} \alpha$ profile does provide some constraints on the $\beta$ exponent.

$\mathrm{He} / \mathrm{H}$ abundances are relatively difficult to constrain in B supergiants since different choices of He I lines yield different He abundances, in part due to the well known 
Table 3. Derived non-LTE stellar and wind parameters. Two values are listed for the mass-loss rate of HD 37128 since we fit the minimum and maximum $\mathrm{H} \alpha$ profiles of Prinja et al. (2004).

\begin{tabular}{|c|c|c|c|c|c|c|c|c|c|c|c|c|c|c|c|}
\hline HD & Name & Sp type & $\begin{array}{r}T_{\text {eff }} \\
\mathrm{kK}\end{array}$ & $\begin{array}{l}\log g \\
\operatorname{cgs}\end{array}$ & $\begin{array}{l}\log g_{\text {eff }} \\
\operatorname{cgs}\end{array}$ & $\log L / L_{\odot}$ & $\begin{array}{l}R_{*} \\
R_{\odot}\end{array}$ & $\begin{array}{r}v_{\infty} \\
\mathrm{km} \mathrm{s}^{-1} \\
\end{array}$ & $v_{\infty} / v_{\mathrm{esc}}$ & $\begin{array}{r}\dot{M} \times 10^{6} \\
M_{\odot} / \mathrm{yr}\end{array}$ & $\begin{array}{c}\beta \\
\mathrm{km} \mathrm{s}^{-1} \\
\end{array}$ & $\begin{array}{c}v \sin i \\
\mathrm{~km} \mathrm{~s}^{-1}\end{array}$ & $\begin{array}{c}\xi \\
\mathrm{km} \mathrm{s}^{-1} \\
\end{array}$ & $\begin{array}{r}M_{V} \\
\mathrm{mag}\end{array}$ & $\begin{array}{c}\dot{M} v_{\infty} R^{0.5} \\
\operatorname{cgs}\end{array}$ \\
\hline 30614 & $\alpha \mathrm{Cam}$ & O9.5 Ia & 29.0 & 3.0 & 2.75 & 5.63 & 26.0 & 1560 & 3.40 & 5.0 & 1.5 & 129 & 30 & -6.6 & 29.40 \\
\hline 37128 & $\epsilon$ Ori & B0 Ia & 27.0 & 2.9 & 2.85 & 5.44 & 24.0 & 1910 & 4.76 & $\begin{array}{r}(\max ) 2.5 \\
(\min ) 2.0\end{array}$ & 1.5 & 91 & 12.5 & -6.3 & $\begin{array}{l}29.17 \\
29.07\end{array}$ \\
\hline 91969 & & B0 Ia & 27.5 & 2.95 & 2.75 & 5.52 & 25.3 & 1470 & 3.33 & 1.0 & 1.5 & 79 & 7.5 & -6.4 & 28.67 \\
\hline 94909 & & B0 Ia & 27.0 & 2.9 & 2.7 & 5.49 & 25.5 & 1050 & 2.55 & 2.0 & 1.5 & $100 \ddagger$ & 10 & -6.4 & 28.62 \\
\hline 122879 & & B0 Ia & 28.0 & 2.95 & 2.7 & 5.52 & 24.4 & 1620 & 3.81 & 3.0 & 1.5 & 92 & 15 & -6.4 & 29.18 \\
\hline 38771 & $\kappa$ Ori & B0.5 Ia & 26.5 & 2.9 & 2.7 & 5.35 & 22.2 & 1525 & 3.84 & 0.9 & 1.5 & 83 & 12.5 & -6.1 & 28.61 \\
\hline 115842 & & B0.5 Ia & 25.5 & 2.85 & 2.65 & 5.65 & 34.2 & 1180 & 3.86 & 2.0 & 1.5 & 84 & 10 & -6.9 & 28.94 \\
\hline 152234 & & B0.5 Ia (N wk) & 26.0 & 2.85 & 2.6 & 5.87 & 42.4 & 1450 & 2.85 & 2.7 & 1.5 & $100 \ddagger$ & 10 & -7.4 & 29.21 \\
\hline 2905 & $\kappa \mathrm{Cas}$ & BC0.7 Ia & 21.5 & 2.6 & 2.45 & 5.52 & 41.4 & 1105 & 2.79 & 2.0 & 2.0 & 91 & 20 & -7.1 & 28.95 \\
\hline 91943 & & B0.7 Ia & 24.5 & 2.8 & 2.6 & 5.35 & 26.3 & 1470 & 3.72 & 0.75 & 1.2 & 79 & 10 & -6.3 & 28.55 \\
\hline 152235 & & B0.7 Ia (N wk) & 23.0 & 2.65 & 2.45 & 5.76 & 47.1 & 850 & 1.98 & 1.25 & 1.5 & 81 & 10 & -7.4 & 28.66 \\
\hline 154090 & & B0.7 Ia & 22.5 & 2.65 & 2.45 & 5.48 & 36.0 & 915 & 2.38 & 0.95 & 1.5 & 78 & 10 & -6.8 & 28.52 \\
\hline 13854 & & B1 Iab & 21.5 & 2.55 & 2.4 & 5.43 & 37.4 & 920 & 2.67 & 0.85 & 2.0 & 97 & 20 & -6.8 & 28.48 \\
\hline 91316 & $\rho$ Leo & B1 Iab (N str) & 22.0 & 2.55 & 2.4 & 5.47 & 37.4 & 1110 & 3.31 & 0.35 & 1.0 & 75 & 10 & -6.8 & 28.17 \\
\hline 148688 & & B1 Ia & 22.0 & 2.6 & 2.4 & 5.45 & 36.7 & 725 & 1.99 & 1.75 & 2.0 & 72 & 15 & -6.8 & 28.68 \\
\hline 14956 & & B1.5 Ia & 21.0 & 2.5 & 2.35 & 5.65 & 50.6 & 500: & 1.33: & 1.0 & 2.0 & $80 \ddagger$ & 10 & -7.4 & 28.35 \\
\hline 152236 & & B1.5 $\mathrm{Ia}^{+}$ & 18.0 & 2.2 & 2.0 & 6.10 & 112.4 & 390 & 1.01 & 6.0 & 2.0 & 74 & 15 & -8.8 & 29.19 \\
\hline 190603 & & B1.5 $\mathrm{Ia}^{+}$ & 18.5 & 2.25 & 1.75 & 5.57 & 59.6 & 485 & 1.62 & 2.5 & 3.0 & 79 & 20 & -7.5 & 28.77 \\
\hline 14143 & & B2 Ia & 18.0 & 2.25 & 2.05 & 5.42 & 52.9 & 645 & 2.21 & 1.05 & 2.0 & 76 & 20 & -7.2 & 28.49 \\
\hline 14818 & 10 Per & B2 Ia & 18.5 & 2.4 & 2.25 & 5.35 & 46.1 & 565 & 1.66 & 0.55 & 2.0 & 82 & 20 & -7.0 & 28.12 \\
\hline 41117 & $\chi^{2}$ Ori & B2 Ia & 19.0 & 2.35 & 2.1 & 5.65 & 61.9 & 510 & 1.43 & 0.9 & 2.0 & 72 & 10 & -7.6 & 28.36 \\
\hline 194279 & & B2 Ia & 19.0 & 2.3 & 2.1 & 5.37 & 44.7 & $550:$ & 1.99: & 1.05 & 2.5 & $80 \ddagger$ & 20 & -7.0 & 28.39 \\
\hline 198478 & 55 Cyg & B2.5 Ia & 16.5 & 2.15 & 1.75 & 5.03 & 40.0 & 470 & 2.02 & 0.23 & 2.0 & 61 & 20 & -6.4 & 27.49 \\
\hline 14134 & & B3 Ia & 16.0 & 2.05 & 1.75 & 5.28 & 56.7 & 465 & 1.94 & 0.52 & 2.0 & 66 & 15 & -7.1 & 28.06 \\
\hline 53138 & $o^{2} \mathrm{CMa}$ & B3 Ia & 15.5 & 2.05 & 1.75 & 5.34 & 65.0 & 865 & 3.25 & 0.36 & 2.0 & 58 & 20 & -7.3 & 28.20 \\
\hline
\end{tabular}

$\ddagger$ : our study requires higher $v \sin i$ values for HD 94909 and HD 152234 than 68 and $76 \mathrm{~km} \mathrm{~s}^{-1}$ obtained by Howarth et al. (1997), respectively, whilst HD 194279 and HD 14956 were not included in their sample.

singlet-triplet problem (Lennon et al. 1991; McErlean et al. 1998). Consequently, we adopt a uniform $\mathrm{He} / \mathrm{H}=0.2$ by number for all our targets. This was selected on the basis of the average N/C enrichment derived relative to Solar values (Sect. 4.2), with respect to recent interior evolutionary models for rotating massive stars (Meynet \& Maeder 2000). Fortunately, an error in $\mathrm{He} / \mathrm{H}$ will only weakly affect the derived stellar temperature and/or mass-loss rates. Indeed, Repolust et al. (2005) have recently obtained a typical $\mathrm{He} / \mathrm{H}$ abundance of 0.2 amongst their sample of Galactic OB supergiants.

CNO abundances were varied to fit the relevant optical lines, with a typical accuracy of $\sim 0.2$ dex. Solar abundances were taken from Grevesse \& Sauval (1998) except oxygen, which was set at $\log (\mathrm{O} / \mathrm{H})+12=8.66$ (Asplund et al. 2004). Abundances of other metallic elements were fixed at Solar values. The primary diagnostic lines used for determination of the nitrogen abundance were N II $\lambda 3995$ and $\lambda 4601-$ 43, plus N III $\lambda 4097$ blend for the earliest subtypes where $\mathrm{N}$ II is weak/absent. Oxygen and carbon abundances were also determined from consideration of the optical lines; O II $\lambda 4069$ 4092 and $\lambda 4590-96$ provided useful constraints on the oxygen abundance, whilst C II $\lambda 4267$ together with C III $\lambda 4647-51$ at early subtypes (blended with O II) were used for carbon. We anticipate accuracies in derived $\mathrm{CNO}$ abundances to be no greater than $\sim 0.15 \operatorname{dex}(\mathrm{O})$ to $\sim 0.3 \operatorname{dex}(\mathrm{C}$ and $\mathrm{N})$, where differences are due to the number of diagnostics available.

\section{Spectroscopic results}

Derived stellar, wind and chemical properties of our sample of OB supergiants based on the intermediate dispersion spectroscopy are provided in Table 3 . We include surface and effective gravities, corrected for the Eddington parameter, $\Gamma_{\mathrm{e}}$, which is the ratio of the radiative acceleration from electron scattering to the stellar gravity. 


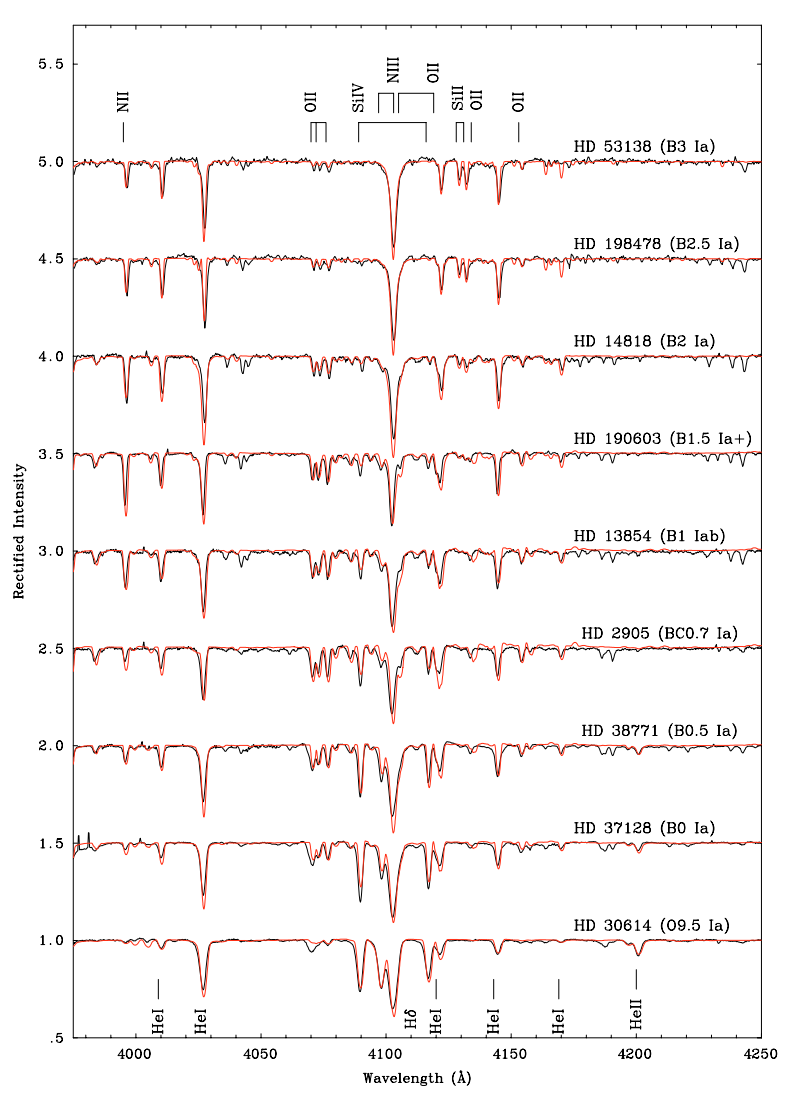

(a)

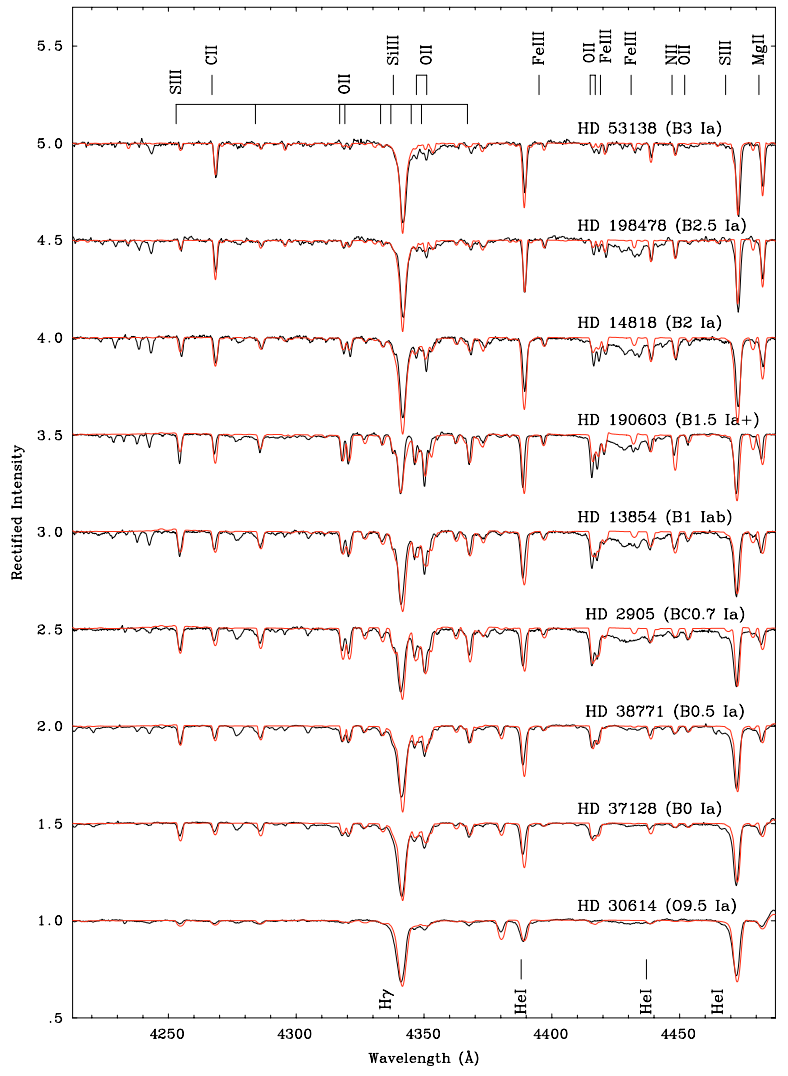

(b)

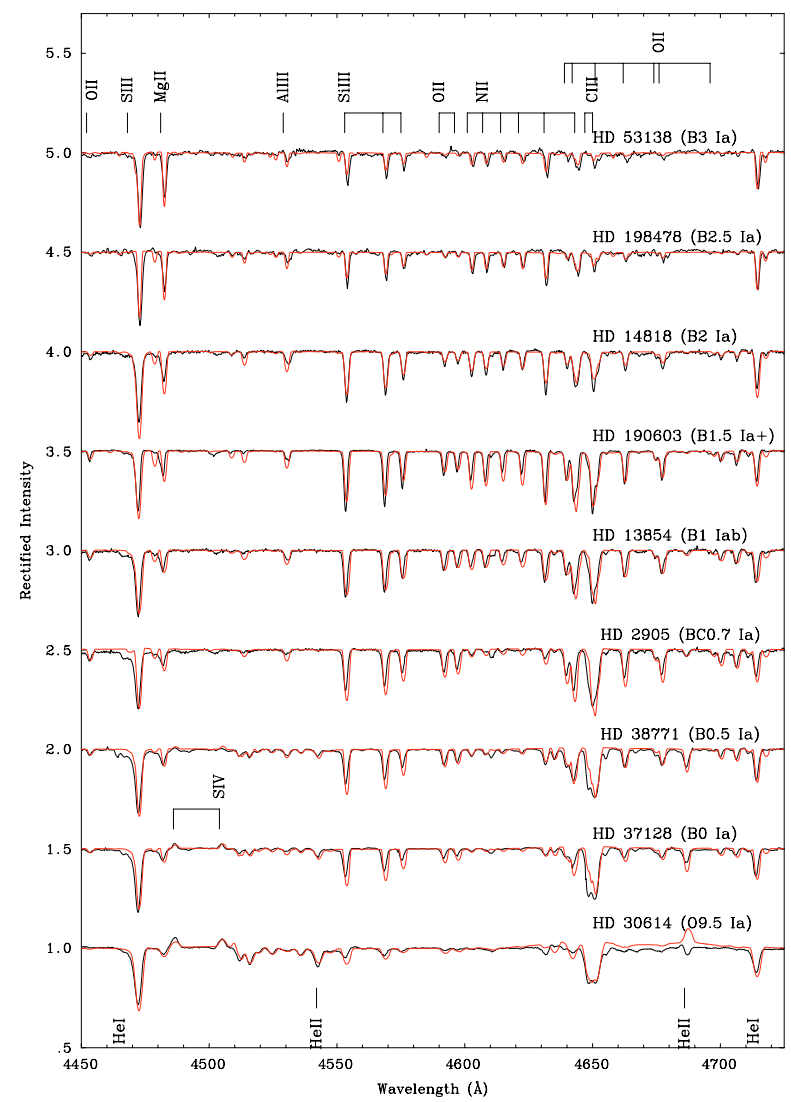

(c)

Fig. 2. a) Synthetic fit (red in electronic version, dotted in paper version) to $\lambda \lambda 3975-4250$ spectral range for representative early B supergiants (solid black). b) Synthetic fit to $\lambda \lambda 4215-4485$ spectral range for representative early B supergiants. c) Synthetic fit to $\lambda \lambda 4450-4725$ spectral range for representative early $\mathrm{B}$ supergiants. 

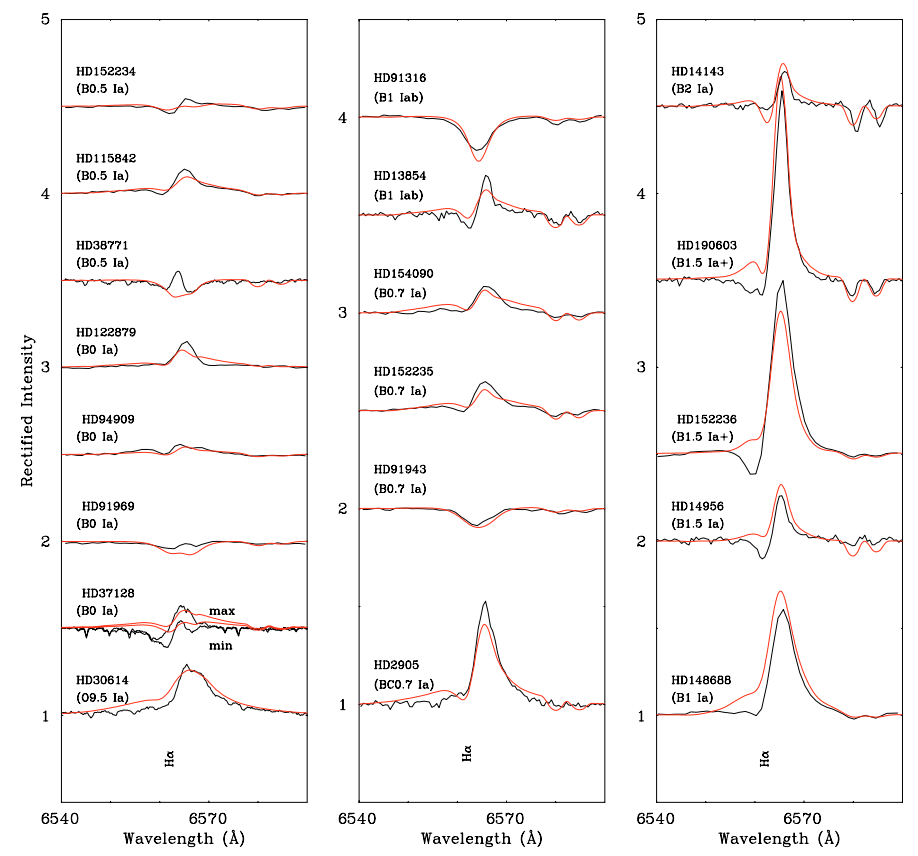

Table 4. Comparison between our derived non-LTE line blanketed temperature scale (in $\mathrm{kK}$ ) of Galactic early B Ia and Iab supergiants and the unblanketed temperature scale of McErlean et al. (1999), plus recent line blanketed Magellanic Cloud results from Trundle et al. (2004), Trundle \& Lennon (2005). For each study the range in temperatures and number of stars studied is indicated. Values for hypergiants are flagged with $*$, and are excluded from our current "mean" temperature scale for Ia supergiants.

\begin{tabular}{|c|c|c|c|c|c|c|c|}
\hline \multirow{2}{*}{$\begin{array}{l}\text { Subtype } \\
\text { O9.5 Ia }\end{array}$} & \multicolumn{2}{|c|}{$\begin{array}{l}\text { McErlean et al. } \\
\text { Galaxy/unblank }\end{array}$} & \multicolumn{2}{|c|}{$\begin{array}{c}\text { Trundle et al. } \\
\text { SMC/blank }\end{array}$} & \multicolumn{2}{|c|}{$\begin{array}{c}\text { This work } \\
\text { Galaxy/blank }\end{array}$} & \multirow{2}{*}{$\begin{array}{r}T_{\text {efl }} \\
\text { mean }\end{array}$} \\
\hline & 32.5 & 1 & & & 29.0 & 1 & \\
\hline O9.7 Ia & 29 & 1 & & & & & 28.5 \\
\hline B0 Ia & 28.5 & 2 & 27 & 1 & $27-28$ & 4 & 27.5 \\
\hline B0.5 Ia & 27.5 & 1 & 27 & 1 & $25.5-26.5$ & 3 & 26 \\
\hline B0.7 Ia & 24 & 1 & & & $21.5-24.5$ & 4 & 22.5 \\
\hline B1 Ia & 23.5 & 1 & $21.5^{*}-25$ & 3 & $21.5-22$ & 3 & 21.5 \\
\hline B $1.5 \mathrm{Ia}$ & $21^{*}-21.5$ & 2 & $20.5-22.5$ & 3 & $18^{*}-21.5$ & 3 & 20.5 \\
\hline B2 Ia & $19-20$ & 4 & 19 & 2 & $18-19$ & 4 & 18.5 \\
\hline B2.5 Ia & 18 & 1 & $16-17$ & 4 & 16.5 & 1 & 16.5 \\
\hline B3 Ia & $17-18.5$ & 4 & 14 & 1 & $15.5-16$ & 2 & 15.5 \\
\hline
\end{tabular}

\subsection{Physical and wind properties}

Figure $2 \mathrm{a}-\mathrm{c}$ compares blue optical spectroscopy for selected O9.5-B3 Ia supergiants with synthetic spectral fits. In general, the quality of spectroscopic fits is excellent, not only for our selected $\mathrm{He}$ or Si diagnostic lines, but also for classification lines such as He I $\lambda 4471$ vs. Mg II $\lambda 4481$, plus weak features of other non-CNO elements, e.g. Al III $\lambda 4529$ and S IV $\lambda 4485-4504$. The major differences reflect weak metal transitions, which are missing from our selected model atoms, with no effect upon the derived physical properties. Apparent discrepancies in the region of $\lambda 4430$ are in fact due to the broad Diffuse Interstellar

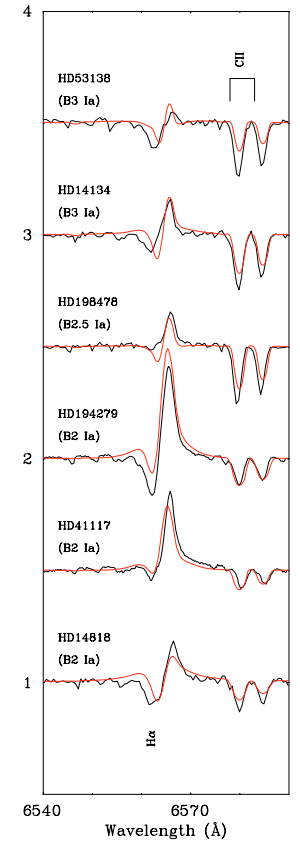

Fig. 3. Spectral fits (red in electronic version, dotted in paper version) to $\mathrm{H} \alpha$ and $\mathrm{C}_{\text {II }} \lambda 6578-82$ for $\mathrm{O} 9.5-\mathrm{B} 3$ supergiants.

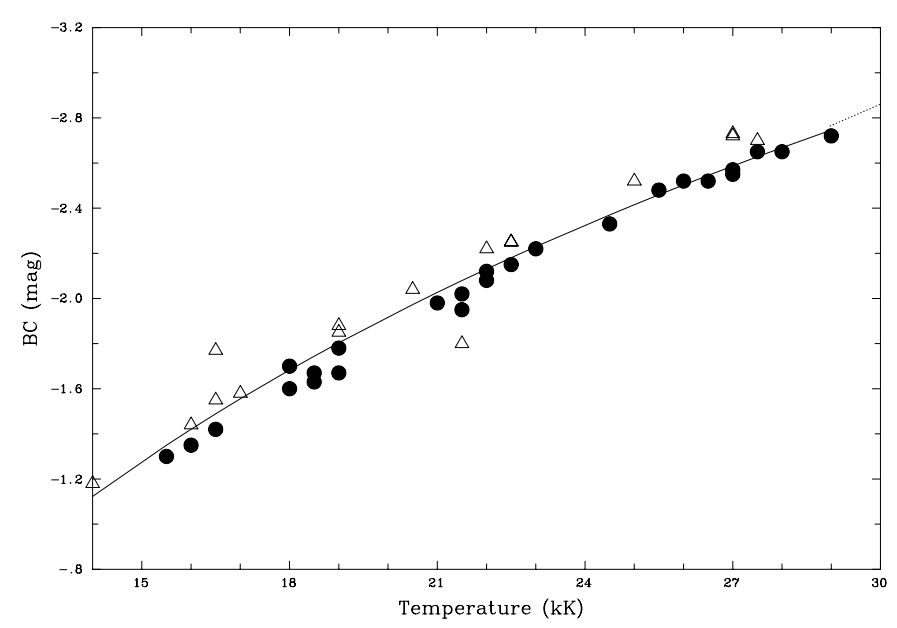

Fig. 4. Bolometric correction (BC) as a function of temperature for Galactic (filled circles, this study) and SMC (open triangles, Trundle et al. 2004; Trundle \& Lennon 2005) B supergiants. The fit to the combined sample (solid line) connects well to the recent Martins et al. (2005) relation for O stars (dotted line).

Band (DIB) at that wavelength, whose strength scales with interstellar reddening. The only clear discrepancies occurs for HD 30614 (O9.5 Ia) and HD 37128 (B0 Ia) at He II $\lambda 4686$ and Si IV $\lambda 4088-4116$.

In Fig. 3 we present spectral fits to all our program stars in the vicinity of $\mathrm{H} \alpha$, with model profiles degraded to the spectral resolution of the individual observations. Overall, agreement is excellent, except that the blue P Cygni absorption component is imperfectly reproduced for the B1-2 supergiants. Note that the C II $\lambda \lambda 6578-82$ doublet is well matched in mid B supergiants in most cases. As already indicated, HD 37128 (B0 Ia) is observed to exhibit large scale $\mathrm{H} \alpha$ variability (Prinja et al. 2004) so we have attempted to reproduce the minimum and maximum emission cases to quantify the range in derived mass-loss rate, namely $2.0-2.5 \times 10^{-6} M_{\odot} \mathrm{yr}^{-1}$. 
Table 4 compares our derived temperatures for B supergiants with those derived previously by McErlean et al. (1999), using hydrostatic, plane-parallel model atmospheres. As with O stars (e.g. Crowther et al. 2002; Repoulst et al. 2004), current line-blanketed, spherically extended model atmospheres indicate a downward revision in the temperature scale, by typically 1-2 kK Indeed, stellar temperatures obtained with CMFGEN seem to be agree well with FASTWIND (Puls et al. 2005), as indicated by Urbaneja (2004) who has used FASTWIND to study HD 37128, HD 38771, and HD 14956 revealing temperatures consistent with our results to $\sim 0.5 \mathrm{kK}$. Given this close agreement, we compare bolometric corrections (BCs) as a function of temperature from the present sample, supplemented by SMC B supergiant results from Trundle et al. (2004) and Trundle \& Lennon (2005) in Fig. 4. A fit to the combined dataset indicates

$\mathrm{BC}=20.15-5.13 \log T_{\text {eff }}$

with a standard deviation of $\sim 0.05 \mathrm{mag}$, which closely matches the recent result for O stars by Martins et al. (2005) at $\sim 30 \mathrm{kK}$.

Spectroscopically estimated masses span a wide range, from $8_{-2}^{+4} M_{\odot}$ (HD 198478) to $76_{-22}^{+44} M_{\odot}$ (HD 152236), based on gravities accurate to $\log g \pm 0.15 \mathrm{dex}$. In general, spectroscopic masses are lower than estimates of the evolutionary masses, indicating another case of a "mass discrepancy", as already established by Trundle et al. (2004) for SMC B supergiants. For example, we estimate an initial (current) evolutionary mass of $20 M_{\odot}\left(18 M_{\odot}\right)$ for HD 198478. In order to resolve the spectroscopic and evolutionary masses, we would require $\log g \sim 2.5$, versus $2.15 \pm 0.15$ obtained for this star.

In Table 5 we compare the present results with those from previous studies, namely (i) Barlow \& Cohen (1977) based on the mid-IR free-free excess technique of Wright \& Barlow (1975) and adopted temperature scale for B supergiants; and (ii) Kudritzki et al. (1999) who adopted the temperature scale of McErlean et al. (1999), based on hydrostatic, planeparallel model atmospheres and derived $\mathrm{H} \alpha$ mass-loss rates with the unblanketed version of FASTWIND (Santolaya-Rey et al. 1997); (iii) Urbaneja (2004) based on FASTWIND (Puls et al. 2005) model atmospheres.

Kudritzki et al. (1999) adopted the Hipparcos distance to HD 38771, whilst we adhere its the membership of Ori OB1c. Putting HD 38771 and the $\mathrm{H} \alpha$ variable HD 37128 aside, consistency between Kudritzki et al. and the present sample is excellent for the other B0-1 supergiants in common, with $\dot{M}_{\mathrm{H} \alpha} / M_{\mathrm{K} 99} \sim 1 \pm 0.1$. In contrast, the present results are systematically higher for B2-3 supergiants, with $\dot{M}_{\mathrm{H} \alpha} / M_{\mathrm{K} 99} \sim 3$, except for HD 53138 for which substantially different absolute magnitudes were adopted in the two studies. This result, together with the good consistency between our results using CMFGEN and those of Urbaneja (2004) based upon the lineblanketed version of FASTWIND (Puls et al. 2005) suggests problems with the application of the unblanketed FASTWIND code to B2-3 supergiants by Kudritzki et al. (1999).

For those 10 stars in common with Barlow \& Cohen (1977), if we adjust their results to our adopted wind velocities and distances, we find ratios $\dot{M}_{\mathrm{H} \alpha} / \dot{M}_{\text {mid-IR }}$ in the range $0.7-2.3$ with an average of 1.4, excluding HD 53138 for which the
Table 5. Comparison between present results for selected B supergiants and those determined from (a) mid-IR free-free excess method of Barlow \& Cohen (1977, BC77), and (b) Kudritzki et al. (1999, K99) following the $\mathrm{H} \alpha$ fitting technique of Santolaya-Rey et al. (1997) plus the plane-parallel hydrostatically derived temperature scale from McErlean et al. (1999). For HD 30614 we compare the present results with both Puls et al. (1996, P96) and the recent line blanketed study of Repolust et al. (2004, R04), whilst we also include line blanketed results from Urbaneja (2004, U04) for HD 37128 and HD 38771. Wind velocities in parenthesis relate to adopted values.

\begin{tabular}{|c|c|c|c|c|c|c|}
\hline HD Sp type & $\begin{array}{r}T_{\text {eff }} \\
\mathrm{kK}\end{array}$ & $\log \left(L / L_{\odot}\right)$ & $\begin{array}{c}v_{\infty} \\
\mathrm{km} \mathrm{s}^{-1}\end{array}$ & $\begin{array}{l}\dot{M} \times 10^{6} \\
M_{\odot} \mathrm{yr}^{-1}\end{array}$ & $\begin{array}{l}M_{V} \\
\mathrm{mag}\end{array}$ & Ref. \\
\hline \multirow[t]{3}{*}{30614 O9.5 Ia } & 30.0 & 5.79 & 1550 & 5.2 & -7.0 & P96 \\
\hline & 29.0 & 5.83 & 1550 & 6.0 & -7.0 & R04 \\
\hline & 29.0 & 5.63 & 1560 & 5.0 & -6.6 & This work \\
\hline \multirow[t]{4}{*}{37128 B0 Ia } & 25.0 & 5.62 & 2300 & 1.7 & -6.8 & BC77 \\
\hline & 28.5 & 5.86 & 1600 & 2.4 & -7.0 & K99 \\
\hline & 27.5 & 5.69 & 1600 & 2.5 & -6.9 & U04 \\
\hline & 27.0 & 5.44 & 1910 & $2-2.5$ & -6.3 & This work \\
\hline \multirow[t]{4}{*}{38771 B0.5 Ia } & 22.0 & 5.30 & 1900 & 0.7 & -6.3 & BC77 \\
\hline & 27.5 & 4.94 & 1350 & 0.27 & -4.8 & K99 \\
\hline & 26.5 & 4.94 & 1350 & 0.48 & -4.9 & U04 \\
\hline & 26.5 & 5.35 & 1525 & 0.9 & -6.1 & This work \\
\hline \multirow[t]{3}{*}{$2905 \mathrm{BC} 0.7 \mathrm{Ia}$} & 20.0 & 5.46 & (1500) & 1.4 & -6.9 & BC77 \\
\hline & 24.0 & 5.70 & 1100 & 2.3 & -7.0 & K99 \\
\hline & 21.5 & 5.52 & 1105 & 2.0 & -7.1 & This work \\
\hline \multirow[t]{2}{*}{13854 B 1 Iab } & 23.5 & 5.53 & 1000. & 0.78 & -6.7 & K99 \\
\hline & 21.5 & 5.43 & 920 & 0.85 & -6.8 & This work \\
\hline \multirow[t]{2}{*}{91316 B1 Iab } & 21.0 & 5.22 & 1580 & 0.86 & -6.4 & $\mathrm{BC} 77$ \\
\hline & 22.0 & 5.47 & 1110 & 0.35 & -6.8 & This work \\
\hline \multirow[t]{2}{*}{14956 B1.5 Ia } & 20.5 & 5.66 & (1200) & 1.2 & -7.0 & U04 \\
\hline & 21.0 & 5.65 & $(500)$ & 1.0 & -7.4 & This work \\
\hline \multirow[t]{2}{*}{$190603 \mathrm{~B} 1.5 \mathrm{Ia}^{+}$} & 19.0 & 5.66 & (1000) & 2.9 & -7.5 & BC77 \\
\hline & 19.5 & 5.63 & 485 & 2.5 & -7.5 & This work \\
\hline \multirow[t]{3}{*}{14143 B2 Ia } & 18.0 & 5.46 & (1000) & 1.2 & -7.1 & BC77 \\
\hline & 20.0 & 5.51 & 650 & 0.30 & -6.95 & K99 \\
\hline & 18.0 & 5.42 & 645 & 1.05 & -7.2 & This work \\
\hline \multirow[t]{2}{*}{14818 B2 Ia } & 20.0 & 5.47 & 650. & 0.25 & -6.9 & K99 \\
\hline & 18.5 & 5.35 & 565 & 0.55 & -7.0 & This work \\
\hline \multirow[t]{3}{*}{41117 B2 Ia } & 18.0 & 5.58 & (1000) & 1.2 & -7.4 & BC77 \\
\hline & 19.5 & 5.70 & 500 & 0.85 & -7.5 & K99 \\
\hline & 19.0 & 5.65 & 510 & 0.9 & -7.6 & This work \\
\hline \multirow[t]{2}{*}{198478 B2.5 Ia } & 15.0 & 5.16 & $(580)$ & 0.56 & -6.8 & BC77 \\
\hline & 16.5 & 5.03 & 470 & 0.23 & -6.4 & This work \\
\hline \multirow[t]{3}{*}{14134 B3 Ia } & 15.0 & 5.24 & $(580)$ & 0.71 & -7.0 & BC77 \\
\hline & 18.0 & 5.48 & 465 & 0.15 & -7.1 & K99 \\
\hline & 16.0 & 5.28 & 465 & 0.52 & -7.1 & This work \\
\hline \multirow[t]{3}{*}{53138 B3 Ia } & 15.0 & 5.28 & 580 & 1.7 & -7.1 & BC77 \\
\hline & 18.5 & 5.22 & 620 & 0.095 & -6.4 & K99 \\
\hline & 15.5 & 5.34 & 865 & 0.36 & -7.3 & This work \\
\hline
\end{tabular}

mid-IR continuum exceeds the $\mathrm{H} \alpha$ result tenfold. Kudritzki et al. (1999) also comment on the peculiar mid-IR brightness 
of HD 53138 with regard to optical and radio mass-loss indices. Overall, agreement is satisfactory between the two techniques. This is of particular interest, given clumping, if present, is predicted to affect these measurements in different ways $\mathrm{H} \alpha$ samples the highest density region immediately above the photosphere, whilst the mid-IR continuum samples free-free emission, superimposed upon the stellar photosphere at larger radii (Runacres \& Owocki 2002). Spectroscopic studies in which clumping is considered generally produce superior spectral fits to far-UV lines (e.g. Evans et al. 2004b). Consequently, a comparison with Barlow \& Cohen (1977) suggests comparable clumping factors in the $\mathrm{H} \alpha$ and mid-IR continuum forming regions.

\subsection{Elemental abundances}

Elemental abundances are presented in Table 6. These represent the first attempt to quantify metal abundances in a substantial sample of Galactic early and mid B supergiants. With respect to current Solar values (Asplund et al. 2005), carbon is depleted by $0.0-0.8 \mathrm{dex}$, nitrogen is enriched by $0.1-1$ dex, with oxygen typically mildly depleted (up to $0.5 \mathrm{dex}$ ). Consequently, the mean $[\mathrm{N} / \mathrm{C}]$ and $[\mathrm{N} / \mathrm{O}]$ ratios for our sample, +1.07 and +0.74 dex respectively, indicate evidence for partial (rotationally mixed?) CNO processing at their surfaces.

These conclusions support the relatively few quantitative abundance estimates for B supergiants from the literature. Previous studies typically relied upon differential abundance analyses (e.g. Smartt et al. 1997) using blanketed LTE model atmospheres (Kurucz 1991), supplemented by non-LTE plane parallel, hydrostatic model atmospheres (e.g. Lennon et al. 1991; Gies \& Lambert 1992). We include results for four morphologically normal B2 Ia supergiants (including HD 14818 and HD 14143) from Lennon (1994), plus two B1-2 Iab/b supergiants (including HD 91316) from Gies \& Lambert (1992) in Table 6. For stars in common between the studies, Gies $\&$ Lambert (1992) obtained $[\mathrm{N} / \mathrm{C}]=+1.11 \mathrm{dex}$ (we derive +1.45 ) for HD 91316 (B1 Iab) and $[\mathrm{N} / \mathrm{C}]=+1.13 \mathrm{dex}$ (versus +0.98 dex here) for HD 198478 (B3 Ia), whilst Lennon (1994) obtained $[\mathrm{N} / \mathrm{C}]=+1.6 \mathrm{dex}$ (we derive $+1.28 \mathrm{dex}$ ) and $[\mathrm{N} / \mathrm{O}]=+0.58$ (versus $+0.78 \mathrm{dex}$ ) for HD $14818(\mathrm{~B} 2 \mathrm{Ia})$, and similar differences for HD 14143 (B2 Ia). Venn (1995) obtained rather lower nitrogen enrichment in a sample of typically lower mass A supergiants and bright giants (see Table 6).

In Fig. 5 we present the observed H-R diagram for our program stars for $[\mathrm{N} / \mathrm{C}]$ and $[\mathrm{N} / \mathrm{O}]$ coded by the degree of enrichment, together with initially rotating $\left(300 \mathrm{~km} \mathrm{~s}^{-1}\right)$ evolutionary models for Solar metallicity from Meynet \& Maeder (2000). In general, greater nitrogen enrichment is observed in lower temperature stars, whilst greater enrichment is predicted at higher luminosity. Note that the lower luminosity mid-B supergiants appear to show greater processing of oxygen than carbon from Fig. 5.

For each of the 20,25 and $40 M_{\odot}$ tracks we present the predicted enrichment at a temperature of $22 \mathrm{kK}$. On average, the predicted $[\mathrm{N} / \mathrm{C}]$ and $[\mathrm{N} / \mathrm{O}]$ ratios of $+0.8-1.3$ and $+0.7-1.2$ match observations rather well, with the highest luminosity star
Table 6. Individual and mean CNO abundances for Galactic B supergiants (given as $\log N(X) / N(\mathrm{H})+12$ ), where $X$ represents the appropriate element, versus Solar abundances (Asplund et al. 2005), to the nearest 0.05 dex. For all stars $\mathrm{He} / \mathrm{H}=0.2$ by number was adopted. In addition, we show the mean non-LTE abundances obtained from two B1-2Ib supergiants from Gies \& Lambert (1992, GL92), four B2Ia supergiant results from Lennon (1994, L94), plus luminosity class II-Ia Galactic A supergiants from Venn (1995, V95).

\begin{tabular}{lllccccc}
\hline \hline HD & Sp type & $T_{\text {eff }} / \mathrm{kK}$ & $\mathrm{C}$ & $\mathrm{N}$ & $\mathrm{O}$ & {$[\mathrm{N} / \mathrm{C}]$} & {$[\mathrm{N} / \mathrm{O}]$} \\
Solar & & & 8.39 & 7.78 & 8.66 & 0.0 & 0.0 \\
\hline 30614 & O9.5 Ia & 29.0 & 8.15 & 8.3 & 8.35 & +0.8 & +0.85 \\
37128 & B0 Ia & 27.0 & 7.95 & 8.15 & 8.55 & +0.8 & +0.5 \\
91969 & B0 Ia & 27.5 & 8.15 & 8.15 & 8.65 & +0.65 & +0.4 \\
94909 & B0 Ia & 27.0 & 7.95 & 8.15 & 8.65 & +0.8 & +0.4 \\
122879 & B0 Ia & 28.0 & 7.95 & 8.15 & 8.65 & +0.8 & +0.4 \\
38771 & B0.5 Ia & 26.5 & 7.95 & 8.25 & 8.55 & +0.9 & +0.6 \\
115842 & B0.5 Ia & 25.5 & 7.80 & 8.25 & 8.45 & +1.1 & +0.7 \\
152234 & B0.5 Ia (N wk) & 26.0 & 7.95 & 8.05 & 8.75 & +0.7 & +0.2 \\
2905 & BC0.7 Ia & 21.5 & 7.9 & 8.0 & 8.75 & +0.7 & +0.1 \\
91943 & B0.7 Ia & 24.5 & 7.65 & 8.15 & 8.4 & +1.1 & +0.6 \\
152235 & B0.7 Ia (N wk) & 23.0 & 7.95 & $8.0:$ & 8.55 & +0.65 & +0.3 \\
154090 & B0.7 Ia & 22.5 & 7.95 & 8.3 & 8.4 & +1.0 & +0.8 \\
13854 & B1 Iab & 21.5 & 7.95 & 8.45 & 8.55 & +1.1 & +0.8 \\
91316 & B1 Iab (N str) & 22.0 & 7.5 & 8.3 & 8.4 & +1.45 & +0.8 \\
148688 & B1 Ia & 22.0 & 7.65 & 8.15 & 8.55 & +1.1 & +0.5 \\
14956 & B1.5 Ia & 21.0 & 7.95 & 8.75 & 8.15 & +1.4 & +1.5 \\
152236 & B1.5 Ia & 18.0 & 7.30 & 8.75 & 8.3 & +2.05 & +1.3 \\
190603 & B1.5 Ia & 18.5 & 7.95 & 8.75 & 8.75 & +1.4 & +0.9 \\
14143 & B2 Ia & 18.0 & 7.6 & 8.7 & 8.6 & +1.75 & +1.0 \\
14818 & B2 Ia & 18.5 & 7.65 & 8.35 & 8.45 & +1.3 & +0.8 \\
41117 & B2 Ia & 19.0 & 7.65 & 8.55 & 8.45 & +1.5 & +1.0 \\
194279 & B2 Ia & 19.0 & 7.95 & 8.65 & 8.45 & +1.3 & +1.1 \\
198478 & B2.5 Ia & 16.5 & 8.25 & 8.25 & 8.3 & +1.0 & +1.2 \\
14134 & B3 Ia & 16.0 & 8.25 & 8.45 & 8.45 & +0.8 & +0.9 \\
53138 & B3 Ia & 15.5 & 7.95 & 8.45 & 8.15 & +1.1 & +1.2 \\
\hline Mean & & & 7.93 & 8.42 & 8.51 & +1.10 & +0.79 \\
\hline GL92 & B1-2 Iab/b & & 7.92 & 8.30 & & +0.99 & \\
L94 & B2 Ia & & & & & +1.81 & +0.65 \\
V95 & A0-F0 & & 8.14 & 8.05 & & +0.52 & \\
\hline
\end{tabular}

HD 152236 revealing the greatest nitrogen enrichment, also as predicted.

Within our sample, all are morphologically normal B supergiants, except that $\kappa$ Cas has a BC subtype (Walborn 1972), suggesting a lower degree of CNO processing. In fact, HD 2905 does have the least enriched $\mathrm{N}$ abundance of our sample (two $\mathrm{N}$ wk supergiants are also only moderately enriched), but does appear to be moderately depleted in $\mathrm{C}$, with no significant processing in O. Consequently, HD 2905 does indeed possess the lowest $[\mathrm{N} / \mathrm{O}]$ and $[\mathrm{N} / \mathrm{C}]$ of our sample, confirming its morphological difference, potentially due to a low initial rotation velocity. 

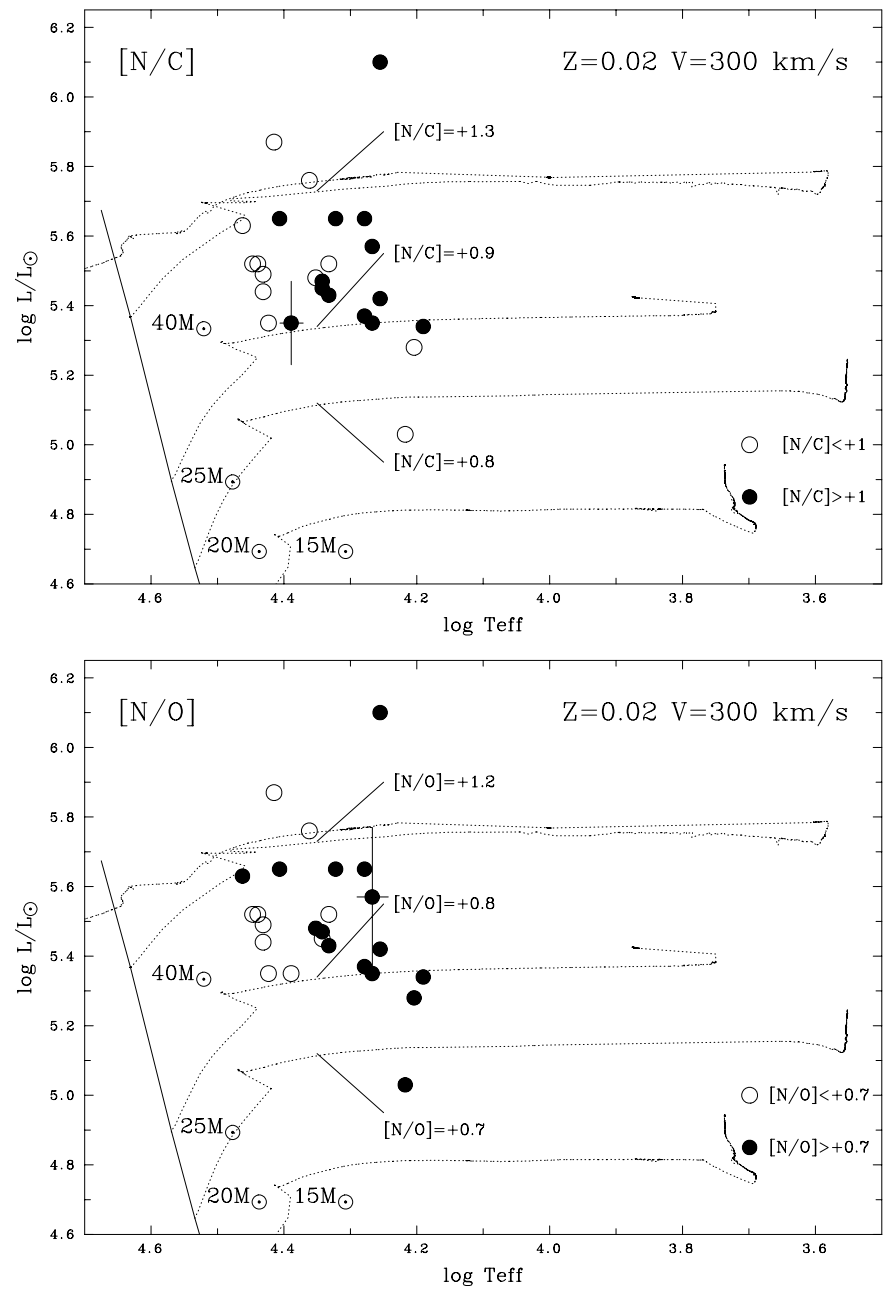

Fig. 5. (Upper panel) Observed H-R diagram for program stars, coded by degree of N/C enrichment, together with tracks from Solar metallicity, rotating $\left(300 \mathrm{~km} \mathrm{~s}^{-1}\right)$ evolutionary models (dotted lines) from Meynet \& Maeder (2000), for which predicted N/C enrichments are indicated at $T_{\text {eff }}=22000 \mathrm{~K}$. The error bar for HD 91943 is indicated ( \pm 0.3 mag uncertainty in distance modulus); (lower panel) as above for N/O. The error bar for HD 190603 is indicated ( \pm 1.0 mag uncertainty in distance modulus).

Finally, Urbaneja (2004) has used FASTWIND to derive physical properties and metal abundances of several Galactic early B supergiants, including HD 37128, HD 38771 and HD 14956. For the B0-0.5 Ia supergiants CNO elemental abundances are consistent at the 0.2 dex level between the two approaches, except that Urbaneja (2004) obtains a $0.25 \mathrm{dex}$ lower $\mathrm{N}$ abundance for HD 37128 and a 0.3 dex lower $\mathrm{C}$ abundance for HD 38771. For HD 14956 (B1.5 Ia), the agreement is poorer - Urbaneja (2004) obtains a 0.55 dex lower $\mathrm{C}$ abundance, 0.15 dex lower $\mathrm{N}$ abundance and 0.4 dex higher $\mathrm{O}$ abundance. Such differences should be taken into account regarding the absolute uncertainty in abundances resulting from such studies.

\section{Wind properties}

Recently, Trundle et al. (2004) and Trundle \& Lennon (2005) have analysed a sample of SMC B supergiants using
FASTWIND, based upon high and intermediate dispersion optical spectroscopy. Before we compare the wind properties of Galactic and SMC B supergiants we wish to investigate whether our use of intermediate dispersion datasets introduces systematic effects on derived stellar properties.

\subsection{Analysis of high resolution observations of HD 115842}

HD 115842 was observed with both the CTIO $1.5 \mathrm{~m} /$ Cassegrain and ESO $2.2 \mathrm{~m} / \mathrm{FEROS}$, allowing us to compare the derived properties of this star from the two datasets. In fact, we were able to match our intermediate dispersion model for HD 115842 to the FEROS echelle dataset, except that the turbulence was increased from $v_{\mathrm{t}}=10 \mathrm{~km} \mathrm{~s}^{-1}$ to $25 \mathrm{~km} \mathrm{~s}^{-1}$. We compare selected regions of the FEROS dataset and synthetic spectrum in Fig. 6, including regions not covered by the CTIO dataset. Overall, the agreement is excellent, such that we are confident in the use of intermediate dispersion for our analysis.

\subsection{Wind momenta for Galactic versus SMC B Supergiants}

We present the wind momenta of our Galactic sample versus luminosity in Fig. 7, separated between early B $(>23 \mathrm{kK})$ and mid $\mathrm{B}(\leq 23 \mathrm{kK})$ subtypes, together with current predictions for early and mid subtypes from Vink et al. (2000). In addition, we include recent Magellanic Cloud results of Evans et al. (2004b), Trundle et al. (2004) and Trundle \& Lennon (2005). The effect of an uncertainty in distance modulus of $\pm 0.5 \mathrm{mag}$ is indicated for HD 38771. This typical uncertainty illustrates why we do not attempt a calibration of the wind momentum luminosity relationship at this time.

For B0-0.5 subtypes, Galactic wind momenta lie close to the Vink et al. (2000) predictions for the majority of cases. In three cases, the derived wind momenta fall 0.5 dex below predictions, namely HD 91943, 115842 and 152234. Due to this spread, there is no clear distinction between the observed wind momenta of early B supergiants in the SMC and Milky Way (compare triangles to circles), although this could be due to small number statistics. If winds of early B supergiants are clumped in the $\mathrm{H} \alpha$ line forming region, we would need to shift the observed wind momenta to smaller values.

For B0.7-3 subtypes, the measured mass-loss rates now lie typically $\sim 0.5$ dex below the calibration of Vink et al., with the exception of HD 2905 (BC0.7 Ia). As above, if $\mathrm{H} \alpha$ derived mass-loss rates of OB stars need to be corrected for clumped winds, as suggested by recent observational evidence (Massa et al. 2003; Evans et al. 2004b), the difference between theory and observation would be exacerbated since the observed data points would move to lower values. In contrast with the case for the earliest B supergiants, we now see a clear separation between Galactic (circles) and SMC (triangles) B0.7-3 supergiants at approximately the predicted offset. 

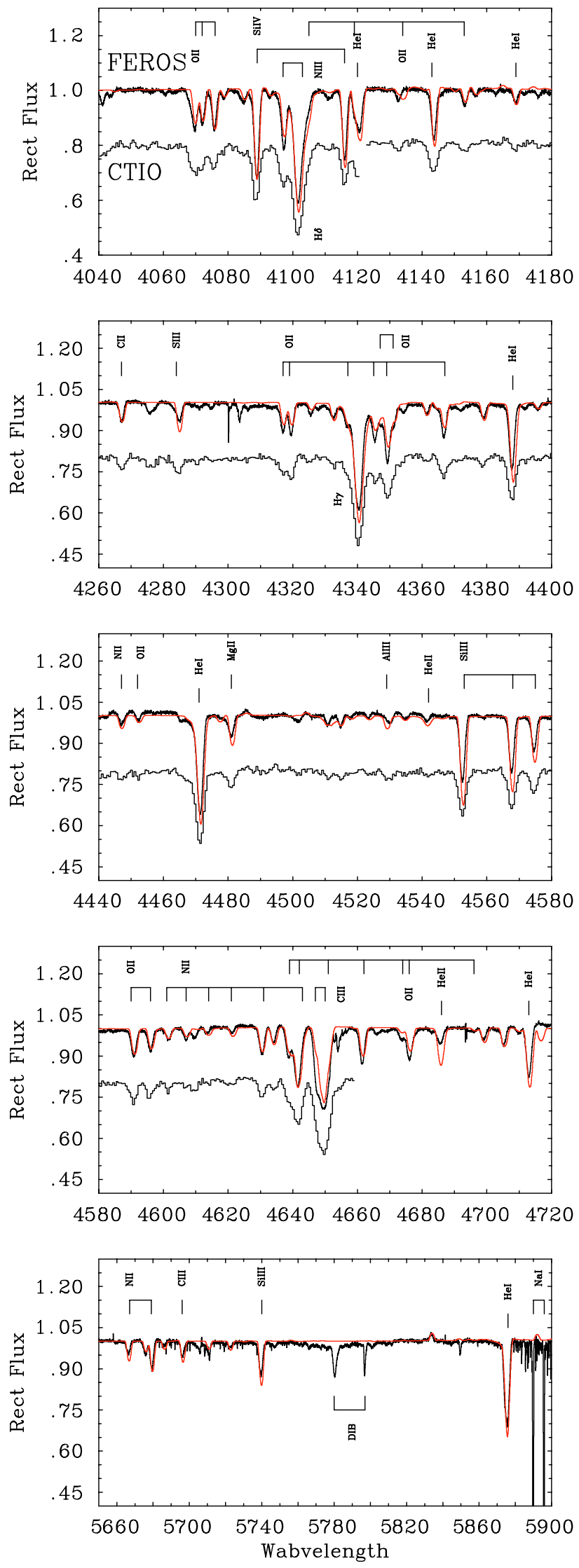

Fig. 6. Spectral comparison between FEROS spectroscopy of HD 115842 (solid black) and synthetic spectroscopy (red in electronic version, dotted in paper version), with CTIO spectroscopy additionally included, offset by -0.2 continuum units.
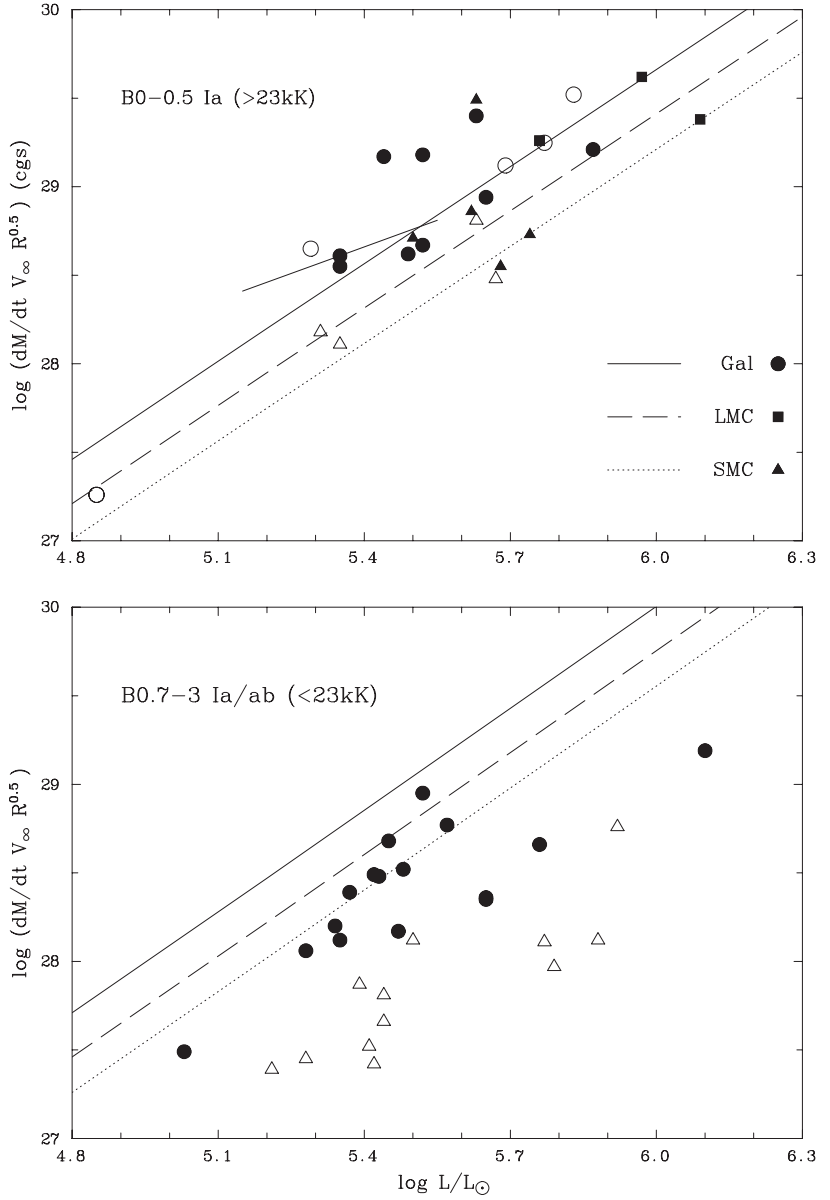

Fig. 7. Comparison between the Galactic B supergiant wind momenta presented here, separated between "early" ( $>23 \mathrm{kK})$ and "mid" ( $\leq 23 \mathrm{kK})$ subtypes, together with other recent studies based on CMFGEN (filled, Evans et al. 2004b) or FASTWIND (open, Trundle et al. 2004; Trundle \& Lennon 2005; Repolust et al. 2004; Herrero et al. 2002), plus radiatively driven wind theory predictions by Vink et al. $(2000,2001)$ for early and mid B supergiants. The effect of an uncertainty of \pm 0.5 mag in distance modulus is indicated for HD 38771 (B0.5 Ia).

\subsection{Bistability jump}

Let us now return to the question of the bistability jump. First, having revised the temperature scale for early B supergiants, let us investigate the sharp $\sim 21 \mathrm{kK}$ bistability jump of Lamers et al. (1995) based on our present results.

In the upper panel of Fig. 8 we repeat the original Lamers et al. (1995) O9.5-B3 supergiant sample, which illustrates the sharp $21 \mathrm{kK}$ jump. This clear step is due to Lamers et al. adopting a single temperature of $20.8 \mathrm{kK}$ for all their seven $\mathrm{B} 1 \mathrm{su}-$ pergiants. In the lower panel of Fig. 8 we present our current results, where we have omitted HD 14956 and HD 194279 for which no reliable wind velocities are known. This sample includes several sources in the original Lamers et al. study, together with values from Evans et al. (2004a), based on measured HST/STIS wind velocities plus model atmosphere results from Evans et al. (2004b), Trundle et al. (2004), Trundle \& Lennon (2005). 

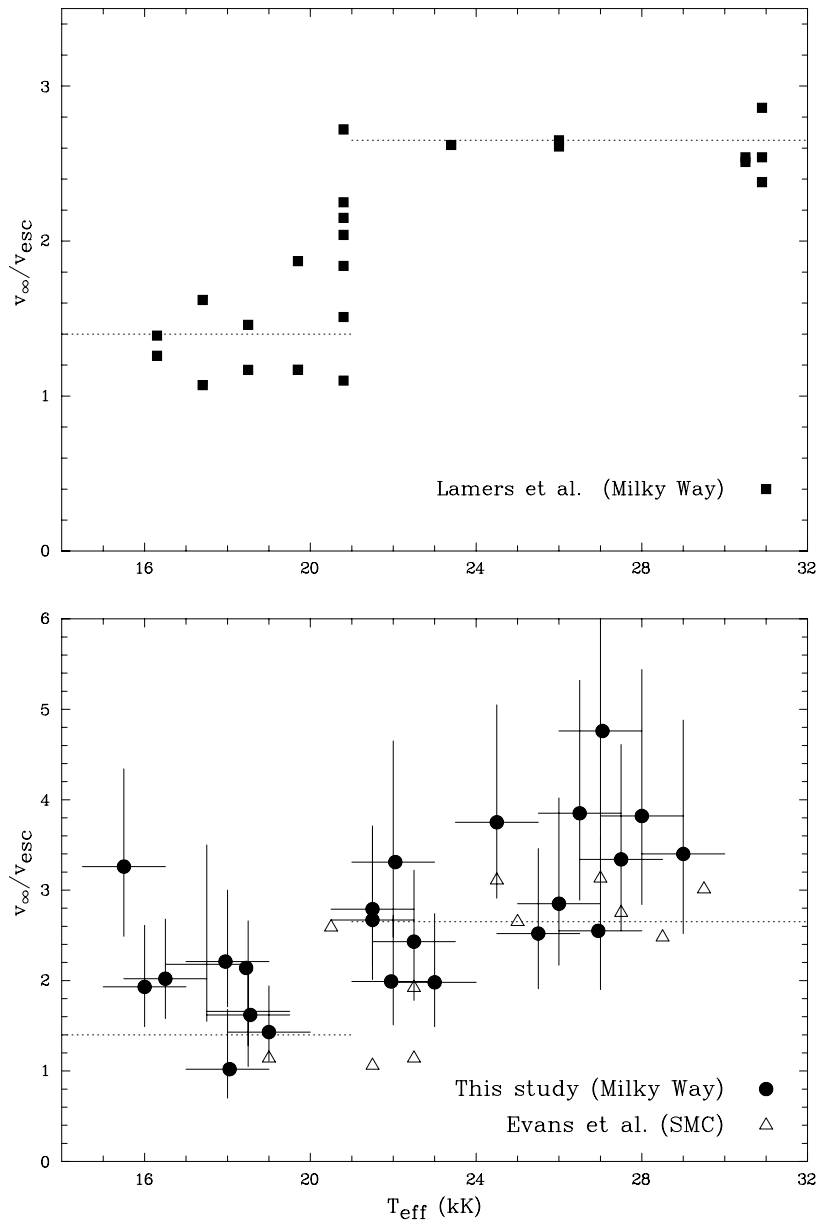

Fig. 8. The ratio $v_{\infty} / v_{\text {esc }}$ as a function of effective temperature for (upper panel) Galactic B supergiants from Lamers et al. (1995) together with the Kudritzki \& Puls (2000) scalings (dotted lines); (lower panel) Galactic B supergiants from the present study (filled circles), together with results from Evans et al. (2004a, open triangles) for SMC B supergiants. Error bars relate to uncertainties in temperature $( \pm 1 \mathrm{kK})$, gravity ( \pm 0.15 to $0.2 \mathrm{dex}$ ) and absolute magnitude ( \pm 0.3 to $1 \mathrm{mag}$ ). We have omitted the two stars (HD 14956, HD 194279) for which reliable wind velocities are not known.

Error bars allow for uncertainties in distance, surface gravity and temperature. Since distances to Galactic early-type supergiants are notoriously imprecise (witness the Hipparcos versus Ori OB1 distance estimates to HD 38771), we provide error bars allowing for uncertainties in distance and surface gravity. Of these, the latter dominates, even for cases with particularly poorly constrained distances, such as HD 190603.

In contrast with the earlier study, there is a gradual downward trend of $v_{\infty} / v_{\text {esc }}$ with temperature, albeit with a large scatter. For $T_{\text {eff }}>24 \mathrm{kK}$ (approximately B0.5 Ia and earlier), $v_{\infty} / v_{\text {esc }} \sim 3.4$, for $20 \mathrm{kK} \leq T_{\text {eff }} \leq 24 \mathrm{kK}$ (approximately B0.7-1 Ia) $v_{\infty} / v_{\text {esc }} \sim 2.5$, and for $T_{\text {eff }}<20 \mathrm{kK}$ (approximately B1.5 Ia and later) $v_{\infty} / v_{\text {esc }} \sim 1.9$. This reveals that the B1 "jump" is misleading in the context of normal B supergiants. Prinja \& Massa (1998) came to similar conclusions based on a larger B supergiant sample, albeit with an adopted subtype-temperature calibration. Amongst the present sample,

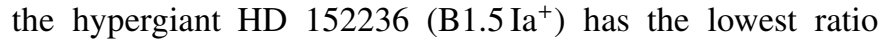

$\left(1.0_{-0.3}^{+0.6}\right)$ whilst HD 37128 (B0 Ia) has the highest ratio of all $\left(4.8_{-1.2}^{+2.0}\right)$.

Vink et al. (1999) predicted a dramatic increase in wind densities of early B supergiants below $\sim 25 \mathrm{kK}$ due to $\mathrm{Fe}$, the dominant line driving ion, shifting to lower ionization stage. This temperature is close to the boundary between B 0.5 and B0.7 subtypes where changes in $v_{\infty} / v_{\text {esc }}$ do occur, although the original Lamers et al. (1995) $21 \mathrm{kK}$ boundary is an equally valid $v_{\infty} / v_{\text {esc }}$ step. However, no significant increase in massloss rate below $\sim 24 \mathrm{kK}$ is identified from our results.

Finally, recall empirical values of the Lamers et al. (1995) "bistability jump" were adopted by Vink et al. (1999, 2000) in their radiatively driven wind calculations. These predictions have subsequently been used in evolutionary calculations (e.g. Meynet \& Maeder 2000) and spectral synthesis calculations (e.g. Rix et al. 2004). Consequently, our re-determination of physical parameters and wind properties of early B supergiants has potential consequences for evolutionary and spectral synthesis calculations.

\subsection{UV morphology}

The primary role of the present study is to provide physical parameters and wind properties of Galactic B supergiants based on optical diagnostics, with reference to the bistability "jump". We shall postpone a detailed discussion of UV spectral comparisons to elsewhere, but wish to comment on one specific aspect relating to UV morphology. Walborn (1971) introduced the B0.7 subtype on the basis of the behaviour of Si III $\lambda 4552 /$ Si IV $\lambda 4089$. Walborn \& Nichols-Bohlin (1987) found that B0.5 Ia and B0.7 Ia supergiants were well defined and quite distinct in their UV spectral morphology, with regard to the presence/absence of $\mathrm{C} \mathrm{II}$ and Al III P Cygni lines, plus the shape of the Si IV absorptions. Between B0.5 and B0.7 the blue-shifted Si IV absorptions suddenly become narrower and deeper, the latter reversing the smooth trend from earlier types.

We have shown that the bistability "jump" does not have a well defined edge, but rather a general trend to lower $v_{\infty} / v_{\text {esc }}$, particularly between B0.5 and B0.7 subtypes. How successful are our models, which are based on optical diagnostics, at reproducing these distinct UV properties?

In Figs. 9a, b we present comparisons between de-reddened IUE (HIRES short wavelength SWP) observations of selected B supergiants and synthetic spectra. The UV spectra of B supergiants are greatly affected by metal blanketing. Indeed, $\sim 95 \%$ of the strong $\left(W_{\lambda} \geq 0.01 \AA\right)$ lines in the $\lambda \lambda 1250-1900$ region of early $\mathrm{B}$ supergiants are due to Fe III-IV transitions. Fe IV dominates the far-UV blanketing at B0, equal numbers of Fe III and Fe IV lines contribute at B0.7, whilst Fe III dominates at later subtypes. The density of spectral lines is so large that there are no clean UV continuum regions, such that our comparisons are between fluxed datasets.

In general, the UV spectra are fairly well reproduced by the optically derived diagnostics, both prominent $\mathrm{P}$ Cygni wind profiles of SiIV, C IV and Al III, and the primarily Fe-blanketed continuum. The most notable exceptions are (i) C IV $\lambda \lambda 1548-51$ for subtypes later than B0.5 for which 

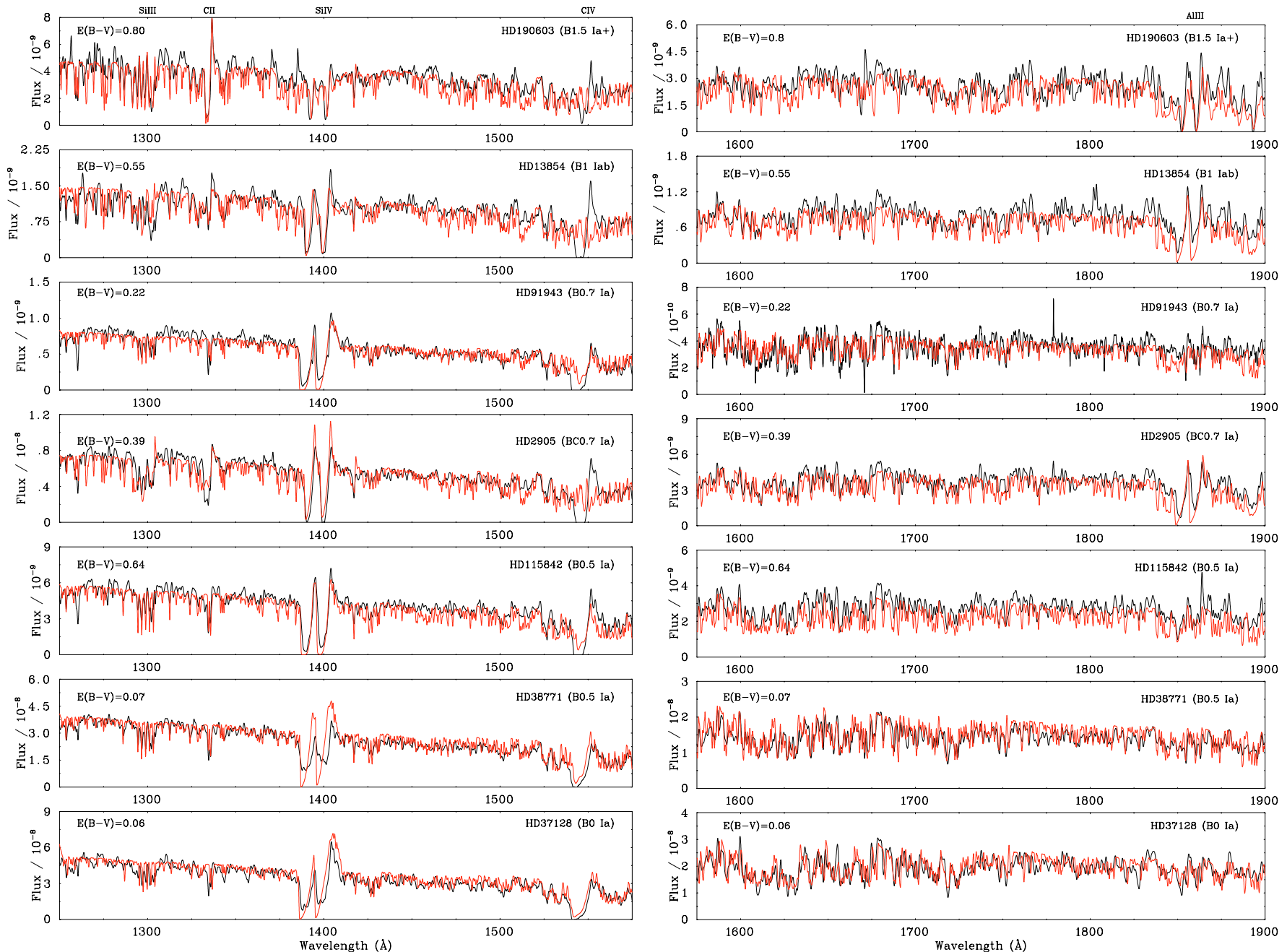

(a)

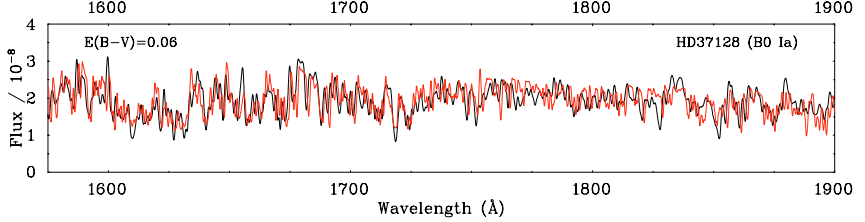

(b)

Fig. 9. a) Comparison between de-reddened HIRES IUE spectroscopy ( $\lambda \lambda 1250-1575)$ of selected early B supergiants (solid black) and synthetic UV spectra resulting from optical analyses (red in electronic version, dotted in paper version). b) Comparison between de-reddened HIRES IUE spectroscopy $(\lambda \lambda 1575-1900)$ of selected early B supergiants (solid black) and synthetic UV spectra resulting from optical analyses (red in electronic version, dotted in paper version).

negligible $\mathrm{P}$ Cygni emission is predicted, in dramatic contrast with observation $-\mathrm{NV} \lambda \lambda 1238-42$ suffers a similar problem for all subtypes - and (ii) The P Cygni absorption strength of Si IV $\lambda \lambda 1393-1402$ is predicted too strong in B0-0.5 supergiants.

Observationally, C IV and C II lines co-exist in B0.7-B2 supergiants, whilst the the former is effectively absent for stellar temperatures below $\sim 25 \mathrm{kK}$. One obvious means of maintaining high ionization states at relatively low stellar temperatures (and hence C IV, N V wind lines) is via X-rays, produced by shocks in the stellar wind, which are also responsible for the clumped winds discussed above. Quantitatively addressing this question is beyond the scope of the present paper, and will be investigated in a future study.

Nevertheless, we also have a partial answer to the reversal of the conventional UV morphologies for two early B supergiants, namely the B0.5 Ia supergiant HD 115842 and B0.7 Ia supergiant HD 91943 (Walborn et al. 1995). In general, B0.5 and B0.7 supergiants are distinct in terms of C II and Al III due to the substantial differences in ionization between $\sim 26 \mathrm{kK}(\mathrm{B} 0.5)$ and $\sim 22.5 \mathrm{kK}$ (B0.7) of these elements. Indeed, the unusually high stellar temperature of $\sim 24.5 \mathrm{kK}$ for HD 91943 (B0.7 Ia) implies a high degree of ionization, such that negligible wind features are predicted, or observed, at $\mathrm{C}$ II and/or Al III. This high temperature results from an unusually low wind density, relative to other B0.7 supergiants, as evidenced by its weak $\mathrm{H} \alpha$ signature in Fig. 3). In contrast, HD 115842 (B0.5 Ia) has a rather strong wind, and so a low stellar temperature, with the Al III feature seen weakly.

Finally, we turn to the other morphological difference between B0.5 and B0.7 supergiants in the UV, i.e. narrower and deeper Si IV absorption at later subtypes. We have demonstrated above that there is indeed a trend for stars with $T_{\text {eff }} \geq$ $24 \mathrm{kK}$ - approximately dividing B0.5 from B0.7 subtypes - to possess higher $v_{\infty} / v_{\text {esc }}$ ratios, i.e. broad, shallow Si IV absorption is observed in B0.5 stars with high wind velocities, with narrow, deep Si IV absorption observed in B0.7 stars with low wind velocities. 
Omitting HD 91943 and HD 115842 from the sample of Howarth et al. (1997), the average B0.5 and B0.7 Ia supergiant wind velocities are $1535 \mathrm{~km} \mathrm{~s}^{-1}$ and $955 \mathrm{~km} \mathrm{~s}^{-1}$, respectively. The wind velocities of the morphologically unusual stars are indeed unusual. HD 91943 (B0.7 Ia) has a fast wind of $1470 \mathrm{~km} \mathrm{~s}^{-1}$, whilst HD 115842 (B0.5 Ia) has a slow wind of $1180 \mathrm{~km} \mathrm{~s}^{-1}$. Of course, a physical explanation is incomplete since the present models fail to predict the observed behaviour of Si IV in cases with fast winds amongst B0-B0.5 supergiants, i.e. all except HD 115842 show too strong Si IV absorption.

Clumping may play a role here too. If the conventional mass-loss diagnostics (e.g. $\mathrm{H} \alpha$ ) in $\mathrm{B}$ supergiants formed in regions where the wind is clumped, the global mass-loss rate will decrease, potentially desaturating Si IV absorption. Similar conclusions were reached by Evans et al. (2004b) in their far-UV study of late $\mathrm{O}$ and early $\mathrm{B}$ supergiants in the Magellanic Clouds, in which the observed S IV $\lambda \lambda 1062-73 \mathrm{ab}-$ sorption could only be matched by clumped models (see also Prinja et al. 2004). A test calculation has been carried out for HD 38771 in which clumping was included, with a volume filling factor of 0.1 . Si IV $\lambda 1393-1402$ was not found to desaturate in this case (C IV $\lambda 1548-51$ did de-saturate), indicating either a higher clumping factor or an alternative explanation.

\section{Conclusions}

We present detailed optical studies of a sample of Galactic B supergiants in order to determine physical parameters and wind properties based on contemporary model atmosphere techniques, for comparison with recent results on Magellanic Cloud B supergiants (e.g. Evans et al. 2004b; Trundle et al. 2004). With respect to Kudritzki et al. (1999) who carried out a similar study based on an adopted temperature scale and a $\mathrm{H} \alpha$ mass-loss determination from an unblanketed code, we obtain lower stellar temperatures (and hence luminosities) and either comparable (early B) or higher (mid B) mass-loss rates. $\mathrm{CNO}$ elements are found to be partially $\mathrm{CNO}$ processed in general. On average, $[\mathrm{N} / \mathrm{C}]$ and $[\mathrm{N} / \mathrm{O}]$ are increased relative to (recently revised) Solar abundances by +1.1 dex and +0.8 dex, respectively. The morphologically unusual B supergiant HD 2905 (BC0.7 Ia) indeed has an unusually low $\mathrm{N}$ enrichment and $\mathrm{C} / \mathrm{O}$ depletion.

With respect to recent theoretical predictions, we find reasonable agreement with those of Vink et al. $(1999,2000)$ for early ( $\geq 23 \mathrm{kK}$ ) B supergiants assuming homogeneous winds, although the predicted winds for later subtype $\mathrm{B}$ are too strong. This would only be exacerbated in the case of clumped winds in the $\mathrm{H} \alpha$ line forming region (Evans et al. 2004b). With respect to recent SMC B supergiant studies, Galactic mid-B supergiants are observed to have systematically stronger winds, although the situation is less clear for early B supergiants. We obtain reasonable agreement between the mid-IR continuum derived mass-loss rates of Barlow \& Cohen (1977) and $\mathrm{H} \alpha$ mass-loss rates suggesting similar clumping factors for these regions, in contrast with expectation (Runacres \& Owocki 2002).

We have investigated the so-called "bistability jump" amongst early B supergiants, in which Lamers et al. (1995) claimed a step in the ratio $v_{\infty} / v_{\text {esc }}$ at $21 \mathrm{kK}$ (B1) from 2.65 to 1.3. From our detailed study of larger sample close to this subtype, we find that there is in fact a gradual decline in $v_{\infty} / v_{\text {esc }}$ from early to mid B supergiants (also identified by Evans et al. 2004a for SMC supergiants) from $\sim 3.4$ above $24 \mathrm{kK}, \sim 2.5$ for $20-24 \mathrm{kK}$, and $\sim 1.9$ below $20 \mathrm{kK}$.

We compare our optically derived spectral fits to UV spectrophotometry of early B supergiants. Morphological differences in the UV between B0.5 ( 26 kK) and B0.7 ( 23 kK) subtypes (Walborn et al. 1995) are attributed to the difference in ionization and wind velocity between these subtypes. Recall Vink et al. (1999) predicted a shift in ionization around $25 \mathrm{kK}$ for B supergiants, due to which wind densities increase dramatically below this temperature. The former does indeed match the observed shift in wind velocities at $\sim 24 \mathrm{kK}$, although no significant increase in mass-loss rate is inferred from our results, affecting evolutionary models which make use of such predictions (e.g. Meynet \& Maeder 2003). Finally, we discuss potential solutions to spectroscopic discrepancies at Si IV (at B0-0.5) and CIV (at B0.7-1.5) which again suggest the presence of shocked, clumped winds in the line forming regions. Further progress requires the development of spectroscopic clumping diagnostics.

Acknowledgements. We wish to thank John Hillier for providing CMFGEN to the general astronomical community. PAC and DJL thank the STScI for financial support from the Director's Discretionary Research Fund where this work was initiated. Southern targets were observed at the CTIO $1.5 \mathrm{~m}$ telescope by Sergio Gonzalez Huerta via the SMARTS Consortium. STScI participation in SMARTS is also funded by the DDRF. We are grateful to Steve Smartt for providing high S/N WHT optical spectroscopy for several targets, and Raman Prinja for providing digital max/min $\mathrm{H} \alpha$ spectroscopy of $\epsilon$ Ori. Chris Evans kindly obtained the FEROS spectrum of HD 115842 on our behalf, to whom we are grateful. Finally, we appreciate useful comments on this paper from Jo Puls and from an anonymous referee.

\section{References}

Asplund, M., Grevesse, N., Sauval, A. J., et al. 2004, A\&A, 417, 751 Asplund, M., Grevesse, N., \& Sauval, A. J. 2005, in Proc. Cosmic abundances as records of stellar evolution and nucleosynthesis, ASP Conf. Ser., ed. F. Bash, \& T. Barnes, in press [arXiv: astro-ph/0410214]

Brown, A. G. A., de Geus, E. J., \& de Zeeuw, P. T. 1994, A\&A, 289, 101

Crowther, P. A. 1997, in Luminous Blue Variables: Massive Stars in Transition, ed. A. Nota, \& H. J. G. L. M. Lamers, ASP Conf. Ser., 120, 51

Crowther, P. A., Hillier, D. J., Evans, C. J., et al. 2002, ApJ, 579, 774 de Zeeuw, P. T., Hoogerwerf, R., de Bruijne, J. H. J., Brown, A. G. A., \& Blaauw, A. 1999, AJ, 117, 354

Evans, C. J., Lennon, D. J., Trundle, C., Heap, S. R., \& Lindler, D. J. 2004a, ApJ, 607, 451

Evans, C. J., Crowther, P. A., Fullerton, A. W., \& Hillier, D. J. 2004b, ApJ, 610, 1021

Gies, D. R., \& Lambert, D. L. 1992, ApJ, 387, 673

Haser, S. M. 1995, Ph.D. Thesis, Univ. Munich

Herrero, A., \& Lennon, D. J. 2004, in Proc IAU Symp. 215, Stellar Rotation, ed. A. Maeder, \& P. R. J. Eenens (San Francisco: ASP), 209 
Herrero, A., Puls, J., \& Najarro, F. 2002, A\&A, 396, 949

Hillier, D. J., \& Miller, D. 1998, ApJ, 496, 407

Hillier, D. J., Lanz, T., Heap, S. R., et al. 2003, ApJ, 588, 1039

Howarth, I. D., Siebert, K. W., Hussain, G. A. J., \& Prinja, R. K. 1997, MNRAS, 284, 265

Hubeny, I., \& Lanz, T. 1995, ApJ, 439, 875

Kendall, T. R., Lennon, D. J., Brown, P. J. F., \& Dufton, P. L. 1995, A\&A, 298, 489

Kudritzki, R.-P., \& Puls, J. 2000, ARA\&A, 38, 613

Kudritzki, R.-P., Puls, J., Lennon, D. J., et al. 1999, A\&A, 350, 970

Kurucz, R. L. 1991, in Precision Photometry: Astrophysics of the Galaxy, ed. A. G. Davis-Philip, A. R. Upgren, \& P. I. Janes (Schenectady: L. Davis Press), 27

Lamers, H. J. G. L. M., Cerruti-Sola, M., \& Perinotto, M. 1987, ApJ, 314,726

Lamers, H. J. G. L. M., Snow, T. P., \& Lindholm, D. M. 1995, ApJ, 455, 269

Lanz, T., \& Hubeny, I. 2003, ApJS, 146, 417

Lennon, D. J. 1994, Space Sci. Rev., 66, 127

Lennon, D. J., Kudritzki, R.-P., Becker, S. T., et al. 1991, A\&A, 252, 498

Lennon, D. J., Dufton, P. L., \& Fitzsimmons, A. 1992, A\&AS, 94, 569

Martins, F., Schaerer, D., \& Hillier, D. J. 2005, A\&A, 436, 1049

McErlean, N. D., Lennon, D. J., \& Dufton, P. L. 1998, A\&A, 329, 613

McErlean, N. D., Lennon, D. J., \& Dufton, P. L. 1999, A\&A, 349, 553

Meynet, G., \& Maeder, A. 2000, A\&A, 361, 159

Meynet, G., \& Maeder, A. 2003, A\&A, 404, 975

Najarro, F., Hillier, D. J., \& Stahl, O. 1997, A\&A, 326, 1117

Pauldrach, A. W., \& Puls, J. 1990, A\&A, 237, 409

Prinja, R. K., \& Massa, D. 1998, in Boulder-Munich II: Properties of Hot Luminous Stars, ed. I. D. Howarth, ASP Conf. Ser., 131, 218

Prinja, R. K., Rivinius, Th., Stahl, O., et al. 2004, A\&A, 418, 727

Prinja, R. K., Massa, D., \& Searle, S. C. 2005, A\&A, 430, L41
Puls, J., Kudritzki, R.-P., Herrero, A., et al. 1996, A\&A, 305, 171 Puls, J., Urbaneja, M. A., Venero, R., et al. 2005, A\&A, 435, 669 Repolust, T., Puls, J., \& Herrero, A. 2004, A\&A, 415, 349

Repolust, T., Puls, J., Hanson, M. M., Kudritzki, R.-P., \& Mokiem, M. R. 2005, A\&A, 440, 261

Rix, S. A., Pettini, M., Leitherer, C., et al. 2004, ApJ, 615, 98

Runacres, M. C., \& Owocki, S. P. 2002, A\&A, 381, 1015

Santolaya-Rey, A. E., Puls, J., \& Herrero, A. 1997, A\&A, 323, 488

Schmidt-Kaler, Th. 1982, in Landolt-Börnstein, Group VI, Vol. 2b, ed. K. Schaifers, \& H. H. Voigt (Springer-Verlag)

Smartt, S. J., Dufton, P. L., \& Lennon, D. J. 1997, A\&A, 326, 763

Smith, L. J. 2005, in Proc The Formation and Evolution of Massive Young Star Clusters, ed. H. J. G. L. M. Lamers, L. J. Smith, \& A. Nota, ASP Conf. Ser., 322, 159

Trundle, C., \& Lennon, D. J. 2005, A\&A, 434, 677

Trundle, C., \& Lennon, D. J., Puls, J., \& Dufton, P. L. 2004, A\&A, 417,217

Urbaneja, M. 2004, Ph.D. Thesis, University of La Laguna (IAC), Spain

Venn, K. A. 1995, ApJ, 449, 839

Vink, J. S., de Koter, A., \& Lamers, H. J. G. L. M. 1999, A\&A, 350, 181

Vink, J. S., de Koter, A., \& Lamers, H. J. G. L. M. 2000, A\&A, 362, 295

Vink, J. S., de Koter, A., \& Lamers, H. J. G. L. M. 2001, A\&A, 369, 574

Walborn, N. R. 1971, ApJS, 23, 257

Walborn, N. R. 1972, AJ, 77, 312

Walborn, N. R., \& Nichols-Bohlin, J. 1987, PASP, 99, 40

Walborn, N. R., Parker, J. Wm., \& Nichols, J. S. 1995, IUE Atlas of B-Type Spectra from 1200 to $1900 \AA$, NASA Reference Publication, 1363 